\title{
CONSTANT $Q$-CURVATURE METRICS ON CONIC 4-MANIFOLDS
}

\author{
HAO FANG AND BIAO MA
}

\begin{abstract}
We consider the constant $Q$-curvature metric problem in a given conformal class on a conic 4-manifold and study related differential equations. We define subcritical, critical, and supercritical conic 4-manifolds. Following Tro91 and CY95, we prove the existence of constant $Q$-curvature metrics in the subcritical case. For conic 4-spheres with two singular points, we prove the uniqueness in critical cases and nonexistence in supercritical cases. We also give the asymptotic expansion of the corresponding PDE near isolated singularities.
\end{abstract}

\section{INTRODUCTION}

In this paper, we study Branson's $Q$-curvature on conic 4-manifolds. First, we give a brief introduction to the study of $Q$-curvature in conformal geometry, especially on 4-manifolds. Then, we discuss conic 4-manifolds. Finally, we present our main results.

Let $\left(M^{4}, g\right)$ be a compact 4 dimensional smooth Riemannian manifold. Branson's $Q$-curvature [BØ91] is defined as

$$
Q_{g}=-\frac{1}{6} \Delta R-\frac{1}{2}|R i c|^{2}+\frac{1}{6} R^{2},
$$

where $R$ and Ric are the scalar curvature and the Ricci curvature tensor of $g$, respectively. Similar to the role of the Gaussian curvature in the surface theory, $Q$ curvature is related to the geometry of 4-manifolds by the following Gauss-BonnetChern formula:

$$
\int_{M} Q d V_{g}=8 \pi^{2} \chi(M)-\int_{M} \frac{1}{4}|W|^{2} d V_{g}
$$

where $W$ is the Weyl tensor.

Let

$$
k_{g}=\int_{M} Q d V_{g}
$$

Since the Weyl tensor is locally conformally invariant, (1.1) implies that $k_{g}$ is a global conformal invariant of $M$. Suppose that $g_{w}=e^{2 w} g$ is another metric in the same conformal class, where $w \in C^{\infty}$. The corresponding $Q$-curvature for $g_{w}$ is given by

$$
P_{g} w+Q_{g}=e^{4 w} Q_{g_{w}}
$$

Part of Hao Fang's work is supported by a Simons Collaboration Grant. Part of Biao Ma's work is supported by Graduate College Summer Fellowship of University of Iowa. 
Here $P_{g}$ is the Paneitz operator

$$
P_{g} w=\Delta_{g}^{2} w+\operatorname{div}\left(\frac{2}{3} R g-2 R i c\right) d w .
$$

An important property of the Paneitz operator is its conformal transformation law. Namely, $P_{g_{w}} u=e^{-4 w} P_{g}$ for $g_{w}=e^{2 w} g$. In [BØ91] BCY92, CY95, the authors have extensively studied the Paneitz operator, $Q$-curvature and their relation to zeta functional determinants of conformally covariant operators. It is worth noting that the Paneitz operator is also a special case of more generally defined GJMS operators, cf. GJMS92.

A natural question in conformal geometry is finding constant $Q$-curvature metrics in a given conformal class. From an analytical point of view, the problem is equivalent to the following

$$
P_{g} w+Q_{g}=c \cdot e^{4 w}
$$

where $c$ is a constant.

We state some important results in this field. Chang and Yang CY95 have established the existence of constant $Q$-curvature metic if the conformal metric class satisfies following conditions:

a) $P_{g}$ is non-negative,

b) Ker $P_{g}=\{$ constants $\}$,

c) $k_{g}<16 \pi^{2}$.

The number $16 \pi^{2}$ comes from a sharp Moser-Trudinger type inequality due to Adams Ada88, which is critical in the arguments of Chang-Yang CY95. We should remark that this existence result covers many cases. In particular, Gursky Gur99 has shown that conditions (a), (b), and (c) are satisfied when (i) Yamabe constant $Y_{g} \geq 0$, (ii) $k_{g} \geq 0$, and (iii) $M$ not conformal to $S^{4}$. If $k_{g}>16 \pi^{2}$, Djadli and Malchiodi DM08 are able to establish the existence of constant $Q$ curvature metrics if $k_{g} \neq 16 l \pi^{2}, l=1,2,3 \cdots$, by a delicate min-max argument. For $k_{g}=16 \pi^{2}$, some existence results are obtained by J. Li, Y. Li and P. Liu[LLL12] under certain additional conditions. In even dimension $n>4$, constant $Q$-curvature problems have been studied in [Ndi07, BFR09, Bre03.

We now introduce conic 4-manifolds. Let $\left(M^{4}, g_{0}\right)$ be a compact smooth Riemannian 4-manifold. The conical singularities are represented by the conformal divisor

$$
D=\sum_{i=1}^{k} p_{i} \beta_{i}
$$

where $p_{i} \in M$ and $0>\beta_{i}>-1$. For simplicity, we assume that $\beta_{1} \leq \beta_{2} \leq \cdots \leq$ $\beta_{k}$. Let $\gamma(x)$ be a function in $C^{\infty}\left(M^{4}-\left\{p_{i}\right\}\right)$ with following local forms: in a neighborhood of $p_{i}$ :

$$
\gamma(x)=\sum_{i=1}^{k} \beta_{i} \log \left(r_{i}\right) \eta_{i}(x)+f_{i}(x),
$$

where $r_{i}=\operatorname{dist}_{g_{0}}\left(x, p_{i}\right)$ is the distance to $p_{i}, f_{i}(x)$ is locally smooth near $p_{i}$, and $\eta_{i}(x)$ is a local cut-off function near $p_{i}$. Consider a singular metric

$$
g_{D}=e^{2 \gamma} g_{0} \text {. }
$$


$g_{D}$ is a metric conformal to $g_{0}$ on $M^{4}-\left\{p_{i}\right\}$ which has a conical singularity at each $p_{i}$. Let $d V_{D}$ be the volume element of $g_{D}$. Let $H^{2}\left(d V_{D}\right)=W^{2,2}\left(d V_{D}\right)$ be the corresponding Sobolev space with respect to the measure $d V_{D}$. We define the conformal class of $g_{D}$

$$
\left[g_{D}\right]:=\left\{g_{w}=e^{2 w} g_{D}: w \in H^{2}\left(d V_{D}\right) \cap C^{\infty}\left(M-\left\{p_{i}\right\}\right)\right\} .
$$

Note $\left[g_{D}\right]$ depends only on $\left(M^{4}, g_{0}\right)$ and $D$. Function $\log \left|x-p_{i}\right|$ is not in $H_{l o c}^{2}\left(d V_{D}\right)$ and neither is $\gamma(x)$. Let $g_{1} \in\left[g_{D}\right]$. We call a 4-tuple $\left(M^{4}, g_{0}, D, g_{1}\right)$ a conic 4manifold. Here $g_{0}$ is called the background metric and $g_{1}$ is called the conic metric. We remark that our definition of conic manifolds can be generalized to general dimensions. We also note here that our definition of conic singularity is in the sense of Reimannian geometry. See, for example, $\mathrm{CC} 96, \mathrm{CC}^{+} 97$. In particular, our definition is different from similar notation that is used in studies of Kähler geometry.

We study the conformal geometry of conic manifolds. For conic 4-manifolds, a corresponding Gauss-Bonnet-Chern formula first proved in BN19] can be stated as follows:

$$
k_{g_{1}}=\int_{M} Q_{g_{1}} d V_{g_{1}}=\int_{M} Q_{g_{0}} d V_{g_{0}}+8 \pi^{2} \sum_{i=1}^{k} \beta_{i} .
$$

We will also give a detailed proof directly following the line of Troyanov Tro91 in Section 3.

In this paper, we study the constant $Q$-curvature metric for conic 4-manifolds. Our motivations are both analytical and geometrical. Analytically, Moser-TrudingerAdams inequality gives the key estimate in BCY92, CY95, DM08. We find out that that Moser-Trudinger-Adams inequality can be generalized under our new setting. Geometrically, the study of stability condition and conic singularities plays a central role in recent developments in Kähler geometry CDS14, CDS15, CDS13, Tia96, Tia15. It is our intention to find a suitable "stability" condition in conformal geometry.

Finally, we describe our new results. For conic 4-manifold $\left(M, g_{0}, D, g_{1}\right)$, we study the existence of a metric $g_{w} \in\left[g_{D}\right]$ such that the $Q$-curvature of $g_{w}$ is a constant, which is equivalent to finding (weak) solutions in $H^{2}\left(d V_{D}\right)=W^{2,2}\left(d V_{D}\right)$ of the equation:

$$
P_{g_{D}} w+Q_{D}=c \cdot \exp (4 w) .
$$

In fact, $H^{2}\left(d V_{D}\right)$ is equivalent to $H^{2}\left(d V_{0}\right)$ space of $g_{0}$ and by Poincaré inequality, the $H^{2}\left(d V_{0}\right)$ norm of $w$ is given by

$$
\|u\|_{H^{2}\left(d V_{0}\right)}^{2}=\int_{M} u P_{g_{0}} u d V_{0}+\|u\|_{L^{2}\left(d V_{0}\right)}^{2},
$$

when conditions (a) and (b) are satisfied, see Section 2.

Our approach to study conic 4-manifolds comes from pioneering works on conic surfaces by Troyanov and others. Historically, Troyanov Tro89 Tro91 systematically studied the prescribed curvature problems on conic surfaces. In particular, he [Tro91 classified conformal metrics on conic Riemann surfaces into three categories: subcritical, critical, and supercritical. He showed that in subcritical cases, there is a unique constant Gaussian curvature metric. By a geometric construction, Luo and Tian LT92 proved that with more than 2 conic points the solution exists 
if and only if in subcritical case. Chen and Li CL95. proved the same results by classifying solutions of the corresponding PDE. Chen and Li also proved that in the critical case, the only solutions are radial symmetric, like an American football. In a recent work, the first named author and Lai described the limiting process when a subcritical metric deforms continuously towards a critical one FL16.

Motivated by Troyanov's classification, we give the following definition.

Definition 1.1. Let $D=\sum_{i=1}^{k} \beta_{i} p_{i}$ and $\beta_{1}=\min \left\{\beta_{i}\right\}$. A conic 4-manifold $\left(M, g_{0}, D, g_{1}\right)$

a) is called subcritical, if $k_{g_{0}}+8 \pi^{2}\left(\sum_{i} \beta_{i}\right)<8 \pi^{2}\left(2+2 \beta_{1}\right)$;

b) is called critical, if $k_{g_{0}}+8 \pi^{2}\left(\sum_{i} \beta_{i}\right)=8 \pi^{2}\left(2+2 \beta_{1}\right)$;

c) is called supercritical, if $k_{g_{0}}+8 \pi^{2}\left(\sum_{i} \beta_{i}\right)>8 \pi^{2}\left(2+2 \beta_{1}\right)$.

Remark 1.2. For general even dimension $n$, similarly, we can define conic $n$-manifold $\left(M^{n}, g_{0}, D, g_{1}\right)$ with $D=\sum_{i=1}^{k} \beta_{i} p_{i}$. Let $\beta_{1}=\min \left\{\beta_{i}\right\}$. Let $Q_{g_{0}}$ be the corresponding $Q$-curvature. Set $k_{g_{0}}=\int_{M} Q_{g_{0}} d V_{g}$. Then, one can define $M$ to be a subcritical conic manifold if $k_{g_{0}}+\gamma_{n}\left(\sum_{i=1}^{k} \beta_{i}\right)<\gamma_{n}\left(2+2 \beta_{1}\right)$, a critical conic manifold if $k_{g_{0}}+\gamma_{n}\left(\sum_{i=1}^{k} \beta_{i}\right)=\gamma_{n}\left(2+2 \beta_{1}\right)$, or a supercritical conic manifold if $k_{g_{0}}+\gamma_{n}\left(\sum_{i=1}^{k} \beta_{i}\right)>\gamma_{n}\left(2+2 \beta_{1}\right)$, where $\gamma_{n}=\frac{(n-1) !\left|S_{n}\right|}{2}$.

In this paper, we primarily consider subcritical cases for general 4-manifolds and critical cases on the sphere $S^{4}$.

Our first result is the following:

Theorem 1.3. Let $\left(M^{4}, g_{0}, D, g_{D}\right)$ be a conic 4-manifold. Suppose that $P_{g_{0}}$ is nonnegative and its kernel contains only constant functions on $\left(M^{4}, g_{0}\right)$. Let $\beta_{1}=$ $\min \left\{\beta_{i}\right\}$. Suppose $k_{g_{0}}+8 \pi^{2}\left(\sum_{i} \beta_{i}\right)<8 \pi^{2}\left(2+2 \beta_{1}\right)$. Then there is a conformal metric $g_{w} \in\left[g_{D}\right]$ represented by $w \in C^{\tau}(M) \cap C^{\infty}\left(M-\left\{p_{i}\right\}\right)$ for $0<\tau<$ $\min \left\{2,4\left(1+\beta_{1}\right)\right\}$ such that $\left(M^{4}, g_{w}\right)$ has constant $Q$-curvature.

Remark 1.4. After the draft of this paper, it was brought to our attention that Hyder-Lin-Wei HLW20] have studied the solutions of the equation (1.9) on spheres. See also Maalaoui [Maa16] for a different approach. Hyder-Lin-Wei [HLW20] give a solvability condition which is equivalent to our subcritical condition on $S^{4}$. Theorem 1.3 also covers non-flat cases. Hence, our results are more general geometrically. Interestingly, we achieve the same solvability condition for standard 4-sphere via different approaches. Inspired by [HLW20, we can apply the same techniques of this paper to prove the existence of constant $Q$-curvature metrics in the subcritical cases defined in Remark 1.2 with two assumptions a) Paneitz operator $P_{g} \geq 0$ and b) $\operatorname{Ker} P_{g}=\{$ constants $\}$. However, it seems difficult to check a) and b) except for conformally flat manifolds.

We summarize our approach to prove Theorem 1.3. In the subcritical case, following [CY95] and Tro91], we study the II functional defined in [BØ91]. For the II functional of a conic 4-manifold, we obtain the coerciveness and hence the existence of a minimizer. We use a conic version of Moser-Trudinger-Adams inequality, which on Euclidean domains has been proved by Lam and Lu [LL11. Following [BCY92] and CY95, we generalize this inequality to general conic manifolds. 
Following works of Troyanov, we also consider solutions of (1.6) with 2 singular points on conic 4 spheres. By a stereographic projection and a conformal transformation, we consider the PDE on the punctured Euclidean space $\mathbb{R}^{4} \backslash\{0\}$,

$$
\Delta^{2} u=e^{4 u},
$$

where $u$ has singularities at 0 and $\infty$. By our definition of conic singularities, $u$ behaves logarithmically at 0 and infinity. Note $-\frac{1}{8 \pi^{2}} \log |x-p|$ is the Green's function for bilaplacian on $\mathbb{R}^{4}$ at $p \in \mathbb{R}^{4}$. In order to emphasize singularities in (1.7), we can equivalently write the following $\mathrm{PDE}$ on $\mathbb{R}^{4}$ :

$$
\Delta^{2} u=e^{4 u}+8 \pi^{2} \beta_{0} \delta_{0},
$$

where $\delta_{0}$ is the Dirac measure at 0 . We further require that as $|x| \rightarrow \infty$,

$$
u(x) \sim-\left(2+\beta_{1}\right) \log |x|,
$$

to indicate the conic singularity at infinity. (1.8) is a special form of the following

$$
\begin{aligned}
\Delta^{2} u(x) & =e^{4 u(x)}+8 \pi^{2} \sum_{i=1}^{k-1} \beta_{i} \delta_{p_{i}}(x), \\
u(x) & \sim-\left(2+\beta_{k}\right) \log |x|,|x| \rightarrow \infty,
\end{aligned}
$$

where $\delta_{p_{i}}$ is the Dirac measure at $p_{i}$. Each solution of (1.9) represents a conic constant $Q$-curvature metric on $S^{4}$ with conformal divisor $D=\sum_{i=1}^{k-1} p_{i} \beta_{i}+\beta_{k} \infty$, where $\infty$ is the north pole on $S^{4}$, due to the stereographic projection.

To study (1.7), we first discuss radial symmetric solutions. Using a cylindrical coordinate, we reduce the PDE to a $4^{\text {th }}$ order ODE.

$$
v^{\prime \prime \prime \prime}(t)-4 v^{\prime \prime}(t)=e^{4 u},
$$

where $t \in \mathbb{R}$. We have the following:

Theorem 1.5. There is a family of solutions $v_{\alpha}$ of (1.10), parametrized by $\alpha=$ $1+\beta>0$ such that $v_{\alpha}^{\prime}(t)$ goes to $\pm \alpha$ as $t$ goes to $\pm \infty$. Differing by a constant and a translation in $t$, these are the only solutions with linear growth at infinity.

We remark that in a paper HMM19 by Hyder, Mancini and Martinazzi, the existence of radial symmetric solutions for constant Q-curvature metric for $S^{n}$, $n \geq 4$ is established, which includes Theorem 1.5 as a special case. We thank a referee for pointing this out. Our proof is independent and different in flavor.

Furthermore, we have the following uniqueness theorem:

Theorem 1.6. A constant Q-curvature 4-sphere with 2-singular points must be a radial symmetric conic sphere. Both singularities have the same index. Under a cylindical coordinate, the metric is given by one of the solutions described in Theorem 1.5 up to a translation.

In the smooth case, the radial symmetry of solutions of (1.7) has been established by Lin Lin98, using the moving plane method. Lin investigates the asymptotic behavior of the solution $u$ and proves that $\Delta u$ has an asymptotic harmonic expansion at infinity. This expansion implies certain monotonicity of $u$ and allows one to initiate moving plane method near infinity. We should mention that Caffarelli, Gidas, and Spruck CGS89 were first to investigate such expansions in order to apply moving plane method to study the semi-linear elliptic equation $-\Delta u=u^{\frac{n+2}{n-2}}$. Since we allow singularities at the origin and at the infinity, we do not expect to have an 
exact expansion like those in Lin Lin98. However, by careful analysis, we establish a similar asymptotic expansion near each singularity. It generalizes our regularity theorem in Theorem 1.3. See details in Section 7.

We intend to investigate critical cases and supercritical cases further. We expect some nonexistence results for constant $Q$-curvature metric in supercritical cases and critical cases with more than 3 singular points. General supercritical cases are more elusive since we have the existence result DM08.

In a recent work [FW19], the first named author and Wei derive a similar criterion for the existence of constant $\sigma_{2}$ curvature metrics on conic 4-manifolds. In particular, they establish the nonexistence result for supercritical cases and uniqueness result for critical cases. Combined with [FW19], our results imply rich conformal geometry of conic manifolds and indicate an interesting direction. In the future, we would like to explore corresponding topics for more general types of singular manifolds.

We organize the paper as follows. In Section 2, we discuss some function spaces and embedding theorems with conical singularities. In Section 3, we derive a GaussBonnet-Chern formula resembling the one for Riemann surfaces. In Section 4, a Moser-Trudinger-Adams type inequality for conic manifolds is established. In Section 5, we give the proof of Theorem 1.3. In Section 6, we study the radial symmetric solutions and prove Theorem 1.5. In Section 7, a detailed asymptotic expansion of solutions near a conical singularity is given. In Section 8, we use the asymptotic expansion from Section 7 to prove Theorem 1.6.

We would like to thank referees for pointing out some previous works BN19] and HMM19, which were unknown to us. We would like to thank Alice Chang and Paul Yang for their interest in this work. The second named author would like to thank Mijia Lai for help and comments. Part of this work was done during the second named author visiting Shanghai Jiaotong University. He would like to thank the hospitality.

\section{Function spaces}

In this section, we discuss several function spaces in our study and list their relations.

Let $\left(M^{4}, g_{0}, D, g_{D}\right)$ be a 4 dimensional conic manifold according to the definitions similar to (1.3) and (1.4) where $D=\sum_{i=1}^{k} \beta_{i} p_{i}$. Likewise, we assume that

$$
g_{D}=e^{2 \gamma(x)} g_{0} \text {. }
$$

$\gamma$ is given by:

$$
\gamma(x)=\sum \beta_{i} \log \left(r_{i}\right) \eta_{i}(x)+f_{i}(x)
$$

where $f_{i}(x)$ is smooth and $\eta_{i}(x)$ is a smooth cutoff function near $p_{i}$. Let $g_{1}=g_{D}$ and $d V_{i}$ be the volume elements of $g_{i}$ for $i=0,1$ respectively. We can define $H^{2}\left(d V_{i}\right)$ norm of a function $u$ in $C^{\infty}(M)$ by

$$
\|u\|_{H^{2}\left(d V_{i}\right)}^{2}=\int_{M}|u|^{2} d V_{i}+\int_{M}\left|\nabla_{g_{i}} u\right|^{2} d V_{i}+\int_{M}\left|\nabla_{g_{i}}^{2} u\right|^{2} d V_{i}
$$

for $i=0,1$. Let $H^{2}\left(d V_{i}\right)$ be the closure of $C^{\infty}(M)$ under the $H^{2}\left(d V_{i}\right)$ norm. Although $g_{1}$ is not smooth, the related $L^{p}$ spaces have certain comparison theorem and $H^{2}\left(d V_{i}\right)$ are actually the same. This is in fact a crucial point in Troyanov's work Tro91]. Besides, the Poincaré inequality and compact embedding theorem 
for Sobolev space are still valid. The following results are similar to those given in Tro91 which concern surfaces.

Proposition 2.1. (Weighted Sobolev inequality) Let $\Omega$ be an open domain in $\mathbb{R}^{4}$ contains 0 . Let $\beta>-1$. Then there is some constant $C(\Omega)$, independent of $p$ such that for any $u \in C_{c}^{2}(\Omega)$,

$$
\left(\int_{\Omega}|u|^{p} \cdot|x|^{4 \beta} d x\right)^{\frac{1}{p}} \leq C(\Omega) p^{\frac{1}{2}} \cdot\|\Delta u\|_{L^{2}(\Omega)}
$$

Proof. See the Appendix in Tro91].

By partition of unity and Proposition 2.1 we have Sobolev's embedding from $H^{2}\left(d V_{i}\right)$ spaces to $L^{p}\left(d V_{i}\right)$ spaces as a natural extension.

Proposition 2.2. (Sobolev's embedding) There is a constant $C$ such that for all $u \in H^{2}\left(d V_{i}\right)$ and $p \in[1, \infty)$, we have $\|u\|_{L^{p}\left(d V_{i}\right)} \leq C \sqrt{p}\|u\|_{H^{2}\left(d V_{i}\right)}$.

Proposition 2.3. ( $L^{p}$ comparison). Let $\alpha=\min \left\{\beta_{i}+1\right\}$ and $\omega=\max \left\{\beta_{i}+1\right\}$. If $p>\frac{q}{\alpha}$, then $L^{p}\left(d V_{0}\right) \subset L^{q}\left(d V_{1}\right)$. If $p>q \omega$, then $L^{p}\left(d V_{1}\right) \subset L^{q}\left(d V_{0}\right)$.

Proof. See [Tro91].

Proposition 2.4. $H^{2}\left(d V_{0}\right)=H^{2}\left(d V_{1}\right)$.

Proof. By Poincaré's inequality, $H^{2}\left(d V_{i}\right)$ norm is given by $\|u\|_{L^{2}\left(d V_{i}\right)}+\|\Delta u\|_{L^{2}\left(d V_{i}\right)}$. We claim that $\left\|\Delta_{g_{0}} u\right\|_{L^{2}\left(d V_{0}\right)}$ and $\left\|\Delta_{g_{1}} u\right\|_{L^{2}\left(d V_{1}\right)}$ are equivalent. In fact, we have that

$$
e^{2 \gamma(x)} \Delta_{g_{1}} u(x)=\Delta_{g_{0}} u(x)+2 \nabla_{g_{0}} u(x) \cdot \nabla_{g_{0}} \gamma(x) .
$$

Observe that, $\left|\nabla_{g_{0}} \gamma(x)\right| \sim \beta_{i}\left|x-p_{i}\right|^{-1}$ at a neighborhood of $p_{i}$ and smooth elsewhere. By the well known Hardy's inequality,

$$
\left\|\nabla_{g_{0}} u(x) \cdot \nabla_{g_{0}} \gamma(x)\right\|_{L^{2}\left(d V_{0}\right)} \leq C\left\|\nabla_{g_{0}}^{2} u\right\|_{L^{2}\left(d V_{0}\right)} .
$$

Hence, $\left\|\Delta_{g_{1}} u\right\|_{L^{2}\left(d V_{1}\right)}<C\left\|\Delta_{g_{0}} u\right\|_{L^{2}\left(d V_{1}\right)}$. The other direction is the same.

We only have to show

$$
\|u\|_{L^{2}\left(d V_{0}\right)} \leq C\|u\|_{H^{2}\left(d V_{1}\right)} \text { and }\|u\|_{L^{2}\left(d V_{1}\right)} \leq C\|u\|_{H^{2}\left(d V_{0}\right)} .
$$

Let $\alpha=\min \left\{\beta_{i}+1\right\}$ and $\omega=\max \left\{\beta_{i}+1\right\}$. By Proposition 2.3 we can choose $p>2 \omega$ to get $\|u\|_{L^{2}\left(d V_{0}\right)} \leq\|u\|_{L^{p}\left(d V_{1}\right)}$. Then we use Proposition 2.2 to get $\|u\|_{L^{2}\left(d V_{0}\right)} \leq$ $C\|u\|_{H^{2}\left(d V_{1}\right)}$. The second inequality in (2.2) can be proved by choosing $p>\frac{2}{\alpha}$.

Since we have established Proposition 2.4, from now on, we do not distinguish $H^{2}\left(d V_{i}\right)$.

The following two propositions are quite standard. See [Tro89] for details.

Proposition 2.5. (compact embedding) The embedding $H^{2}\left(d V_{i}\right) \hookrightarrow L^{p}\left(d V_{i}\right)$ for $i=0,1$ is compact for $1<p<\infty$.

Proposition 2.6. ( Poincaré's inequality) If $\int_{M} v d V_{i}=0, i=0,1$, then $\|v\|_{H^{2}} \leq$ $C\|\Delta v\|_{2}$ for some constant $C=C(M)$. 


\section{Gauss-Bonnet-Chern formula}

In this section, we establish the conformal Gauss-Bonnet-Chern formula (1.5), which is originally proved in BN19. Our discussion follows the approach of Troyanov Tro91 and notations in previous sections.

On $\left(M^{4}, g\right)$, recall that the Gauss-Bonnet-Chern formula

$$
\int_{M} Q(x) d V_{g}(x)+\frac{1}{4} \int_{M}|W(x)|^{2} d V_{g}(x)=8 \pi^{2} \chi(M) .
$$

In this section, we will describe the contribution of conical singularities to this Gauss-Bonnet-Chern formula.

Let $\left(M^{4}, g_{0}, D, g_{D}\right)$ be a conic 4-manifold and $g_{1}=g_{D}$. A good choice of base metric $g_{0}$ will simplify the discussion. Thus we use the conformal normal coordinates by [LP87]. We can find a metric $g \in\left[g_{0}\right]$ such that around each given point $p_{i}$, the normal coordinates of $g$ satisfy

$$
\operatorname{det}(g(x))=1+O\left(|x|^{N}\right)
$$

for any $N \in \mathbb{N}$.

Lemma 3.1. Suppose that $B_{\epsilon}(x)$ is a ball with radius $\epsilon$ centered at $x \in M$. Let $\delta$ be the injective radius of $x \in M$ Let $h(x, y)=\log (|x-y|) f(y)$ where $f(y)$ is a smooth function supported in $B_{\delta}(x)$ and equals 1 in $B_{\epsilon}(x)$. Then

$$
\lim _{\epsilon \rightarrow 0} \int_{M \backslash B_{\epsilon}(x)} P_{y}(h(x, y)) d V_{g}(y)=8 \pi^{2}=4\left|S^{3}\right|,
$$

where $P_{y}$ is the Paneitz operator with respect to $y$ and $\left|S^{3}\right|$ is the volume of a unit 3-sphere.

Proof. Let $\left(r, \theta^{i}\right)$ be the normal coordinates at $x$. Let $f \in C^{2}(M-\{x\})$. If $f=f(r)$ then

$$
\Delta f(r)=f^{\prime \prime}+\frac{3}{r} f^{\prime}+f^{\prime} \partial_{r} \log \sqrt{\operatorname{det}(g)},
$$

where $f^{\prime}$ denotes the derivative with respect to $r$. In particular, $\Delta \log r=\frac{2}{r^{2}}+$ $\frac{1}{2 r} \partial_{r} \log \operatorname{det}(g)$. Suppose that $P u=\Delta^{2} u+\operatorname{div}\left(A_{g} d u\right)$ where $A_{g}=\frac{2}{3} R g-2 R i c$. Divergence theorem then gives

$$
\begin{aligned}
\int_{M \backslash B_{\epsilon}(x)} P_{y}(h(x, y)) d V_{g}(y) & =-\int_{\partial B_{\epsilon}(x)}\left(\frac{\partial}{\partial r} \Delta \log r+O\left(r^{-2}\right)\right) d \Omega_{g} \\
& =\int_{\partial B_{\epsilon}} \frac{4}{\epsilon^{3}} d \Omega_{g}+o(1),
\end{aligned}
$$

since $\partial_{r} \Delta \log r=-\frac{4}{r^{3}}+O\left(r^{N-3}\right)$ by our assumption (3.1). Take $\epsilon \rightarrow 0$ then the right hand side of (3.2) approaches $8 \pi^{2}$ since the unit sphere $S^{3}$ has volume $2 \pi^{2}$.

Proposition 3.2. (Gauss-Bonnet-Chern) Suppose that $g_{1}=e^{2 \gamma} g_{0}$ is the metric with $k$ singular points given by divisor $D=\sum_{i=1}^{k} p_{i} \beta_{i}$, where $p_{i} \in M, \beta_{i}>-1$. Suppose that $\gamma(x)=\beta_{i} \eta_{i} \log r+f(x)$ in a neighborhood of $p_{i}, r=\operatorname{dist}\left(p_{i}, x\right)$ and $f(x) \in H^{2}(M) \cap C^{\infty}\left(M-\left\{p_{i}\right\}\right)$. Then we have the formula

$$
\int_{M} Q_{g_{1}} d V_{g_{1}}=\int_{M} Q_{g_{0}} d V_{g_{0}}+8 \pi^{2}\left(\sum_{i=1}^{k} \beta_{i}\right)
$$


Proof. First, we assume that $f$ is $C^{\infty}(M)$. Observe that

$$
\begin{aligned}
\int_{M} Q_{g_{1}} d V_{g_{1}} & =\int_{M} e^{-4 \gamma}\left(P_{g_{0}} \gamma+Q_{g_{0}}\right) d V_{g_{1}} \\
& =\int_{M}\left(P_{g_{0}} \gamma+Q_{g_{0}}\right) d V_{g_{0}} .
\end{aligned}
$$

We need to compute $\int_{M} P_{g} \gamma d V_{g}$. Suppose that $\epsilon$ is a positive real number smaller than the injective radius at each $p_{i}$. Let $B_{i}=B_{\epsilon}\left(p_{i}\right)$ be the ball with radius $\epsilon$ at $p_{i}$. We consider the integral $\int_{M-\cup B_{i}} P_{g_{0}} \gamma d V_{g_{0}}$. By divergence theorem, we see that this integral is just

$$
\sum_{i=1}^{k} \int_{\partial B_{i}}\left(\frac{\partial}{\partial n}\left(\Delta_{g_{0}} \gamma\right)+\left\langle n, A_{g_{0}}(\nabla \gamma)\right\rangle\right) d \Omega_{g_{0}},
$$

where $d \Omega_{g_{0}}$ is the area element on the geodesic sphere. Note $\gamma(x)=f(x)+$ $\beta_{i} \eta_{i} \log (r)$ near $p_{i}$. Thus, by Lemma 3.1, we see that

$$
\lim _{\epsilon \rightarrow 0} \int_{M-\cup B_{\epsilon}\left(p_{i}\right)} P_{g_{0}} \gamma d V_{g_{0}}=\sum_{i=1}^{k} 8 \pi^{2} \beta_{i} .
$$

For more general $f \in H^{2}(M) \cap C^{\infty}\left(M-\left\{p_{i}\right\}\right)$, let $\phi_{\epsilon}$ be a smooth function such that $\operatorname{supp}\left(1-\phi_{\epsilon}\right) \subset \cup_{i=1}^{k} B_{2 \epsilon}\left(p_{i}\right)$ and $\phi_{\epsilon}=0$ on $B_{\epsilon}\left(p_{i}\right)$. We can assume that $\left|D^{k} \phi_{\epsilon}\right|<C \epsilon^{-k}$ for $k=1,2,3,4$. Now it suffices to prove

$$
\lim _{\epsilon \rightarrow 0} \int_{M} \phi_{\epsilon} P_{g_{0}} f d V_{g_{0}}=0 \text {. }
$$

Let $B_{\epsilon}=\cup_{i} B_{\epsilon}\left(p_{i}\right)$. Note that the highest order term of $P_{g_{0}}$ can be estimated by $H_{\text {ölder' }} s$ inequality

$$
\begin{aligned}
\left|\int_{M} \Delta f \Delta \phi_{\epsilon} d V_{g_{0}}\right| & \leq\left|\int_{B_{2 \epsilon}} C \epsilon^{-2} \Delta f d V_{g_{0}}\right| \\
& \leq C\left|\int_{B_{2 \epsilon}} \epsilon^{-4} d V_{g_{0}}\right|^{\frac{1}{2}}\left|\int_{B_{\epsilon}}(\Delta f)^{2} d V_{g_{0}}\right|^{\frac{1}{2}} \\
& \leq C\|f\|_{H^{2}\left(B_{\epsilon}\right)} \rightarrow 0 \quad \text { as } \epsilon \rightarrow 0 .
\end{aligned}
$$

The lower order terms can be estimated by

$$
\begin{aligned}
\int_{M} \phi_{\epsilon} \operatorname{div}\left(A_{g_{0}} d f\right) d V_{g_{0}} & =\int_{M} A_{g_{0}}\left(\nabla \phi_{\epsilon}, \nabla f\right) d V_{g_{0}}=\int_{B_{2 \epsilon}} A_{g_{0}}\left(\nabla \phi_{\epsilon}, \nabla f\right) d V_{g_{0}} \\
& \leq \int_{B_{2 \epsilon}}|| A_{g_{0}} \|_{\infty} \cdot\left|\nabla \phi_{\epsilon}\right| \cdot|\nabla f| d V_{g_{0}}
\end{aligned}
$$

By Hölder's inequality, the lower order terms go to 0 as $\epsilon$ goes to 0. (3.4) and (3.5) proves (3.3) which concludes the whole proof.

For later use, we state the following corollary.

Corollary 3.3. Suppose that in a neighborhood of $p_{i}, \gamma(x)=\beta_{i} \eta_{i} \log (r)+f(x)$ for some smooth $f(x)$. Then $Q_{1} e^{4 \gamma} \in L^{p}\left(d V_{0}\right)$ for $1<p<2$ and $Q_{1} \in L^{2}\left(d V_{1}\right)$.

Proof. Recall $P_{g} v=\Delta_{g}^{2} v+\operatorname{div}\left(A_{g} d v\right)$. As in the definition (1.3) in a neighborhood of $p_{i}$,

$$
\gamma(x)=\beta_{i} \log (r)+f(x),
$$


for some smooth $f(x)$. By calculation in Lemma 3.1, we see that,

$$
\Delta_{g_{0}}^{2} \gamma(x)=O\left(r^{N-4}\right) \text {. }
$$

Recall the leading term of $P_{g_{0}}$ is $\Delta_{g_{0}}^{2}$. Therefore, we obtain from (3.6) that

$$
\left|P_{g_{0}} \gamma\right| \leq C r^{-2} \text {. }
$$

Thus, we conclude from (3.6) that

$$
Q_{1} e^{4 \gamma}=\left(P_{g_{0}} \gamma+Q_{0}\right) \sim O\left(r^{-2}\right),
$$

which implies that $Q_{1} e^{4 \gamma} \in L^{p}\left(d V_{0}\right)$ for $1<p<2$. Moreover, we write (3.8) as $Q_{1} \sim O\left(r^{-2-4 \beta_{i}}\right)$, then

$$
Q_{1}^{2} e^{4 \gamma} \sim O\left(r^{-4-4 \beta_{i}}\right)
$$

Since $-4-4 \beta_{i}>-4$, we have $Q_{1} \in L^{2}\left(d V_{1}\right)$.

\section{A Modified Moser-Trudinger-Adams Inequality}

In this Section, we establish a conic Moser-Trudinger-Adams inequality.

A singular version of Moser-Trudinger-Adams inequality on bounded domains in Euclidean spaces has been proved by Lam and Lu LL11. In this section, we first give a quick proof based on a comparison principle of Talenti Tal76] and a lemma by Tarsi Tar12. Then we establish a corresponding inequality for general conic 4-manifolds.

We first introduce Talenti's comparison principle. Let $f$ be a measurable function with support in a bounded domain $\Omega \subset \mathbb{R}^{n}$. Let $\lambda(s)=m(\{x:|f(x)|>s\})$ and $f^{*}(t)=\sup \{s>0: \lambda(s)>t\}$. The spherical rearrangement $f^{\#}(x)$ of $f$ is defined to be

$$
u^{\#}(x)=u^{*}\left(\omega_{n}|x|^{n}\right), x \in \Omega^{\#} .
$$

Here $\Omega^{\#}$ is a open ball in $\mathbb{R}^{n}$ with the same measure as $\Omega, \omega_{n}$ is the volume of a unit $n$ dimensional ball. Let

$$
b_{n, 2}=\frac{1}{\omega_{n}}\left[\frac{4 \pi^{\frac{n}{2}}}{\Gamma\left(\frac{n}{2}-1\right)}\right]^{\frac{n}{n-2}},
$$

following Ada88. Note that $\omega_{n}=\pi^{n / 2} / \Gamma\left(\frac{n}{2}+1\right)$. So we have $b_{n, 2}=\left[\omega_{n}^{\frac{2}{n}} n(n-\right.$ $2)]^{\frac{n}{n-2}}$.

Lemma 4.1 (Talenti's principle). If $u, v$ are solutions of the following equations,

$$
\left\{\begin{array} { l l } 
{ \Delta u ( x ) = f ( x ) } & { x \in \Omega , } \\
{ u ( x ) = 0 } & { x \in \partial \Omega , }
\end{array} \quad \left\{\begin{array}{ll}
\Delta v(x)=f^{\#}(x) & x \in \Omega^{\#}, \\
v(x)=0 & x \in \partial \Omega^{\#},
\end{array}\right.\right.
$$

then we have that

$$
v \geq u^{\#} \text {. }
$$

We apply Talenti's principle to a $C^{2}$ function $u$ with support in $\Omega$. By definition, $u^{\#}$ is non-increasing. Therefore, if we fix $b>0$ and $-1<\beta<0$, we have

$$
\begin{aligned}
\int_{\Omega} e^{b u^{\frac{n}{n-2}}}|x|^{n \beta} d x & \leq \int_{\Omega^{\#}} e^{b\left(u^{\#}\right)^{\frac{n}{n-2}}}|x|^{n \beta} d x+C(\Omega) \\
& \leq \int_{\Omega^{\#}} e^{b v^{\frac{n}{n-2}}}|x|^{n \beta} d x+C(\Omega),
\end{aligned}
$$


where $v$ comes from (4.2). Thus, in order to prove the modified Moser-TrudigerAdams inequality, we only have to consider spherical symmetric domains and functions.

We then state a lemma of Tarsi Tar12].

Lemma 4.2. Tar12. Let $p>1$. For any $r>0$ there is a constant $C=C(p, r)$ such that for any positive measurable function $f(s)$ on $(1,+\infty)$, satisfying

$$
\int_{1}^{\infty} f^{p} s^{2 p-1} d s \leq 1
$$

then

$$
\int_{1}^{\infty} e^{r F^{q}(t)} \frac{d t}{t^{r+1}} \leq C
$$

where $\frac{1}{p}+\frac{1}{q}=1$, and

$$
F(t)=\int_{1}^{t} \int_{\tau}^{\infty} f(s) d s d \tau
$$

We now prove the following Moser-Trudinger-Adams inequality in bounded Euclidean domains, which first appears in LL11.

Theorem 4.3. Suppose that $\Omega \subset \mathbb{R}^{n}$ is a bounded domain and $0 \in \Omega$. Suppose $u \in C_{c}^{2}(\Omega)$ and $-1<\beta<0$. There is a $C=C(\Omega)$ such that if $\|\Delta u\|_{n / 2} \leq 1$ then

$$
\int_{\Omega} \exp \left(b_{n, 2}(1+\beta)|u|^{\frac{n}{n-2}}\right)|x|^{n \beta} d x \leq C .
$$

The coefficient $b_{n, 2}(1+\beta)$ here is sharp in the sense that the inequality fails for bigger constant. In particular, $b_{4,2}=32 \pi^{2}$.

Proof. By (4.3), we only have to consider radial functions. Suppose that $v(x)$ is a $C^{2}$ radially decreasing function with support in ball $B_{R}$. Let

$$
w(t)=n^{\frac{2}{n}} \omega_{n}^{\frac{2}{n}}(n-2)^{2-\frac{2}{n}} v\left(R t^{-1 /(n-2)}\right) .
$$

We see that

$$
\int_{B_{R}} \exp \left(b_{n, 2} \alpha|v|^{\frac{n}{n-2}}\right)|x|^{n \beta} d x=\frac{n \omega_{n} R^{n \alpha}}{n-2} \int_{1}^{\infty} \exp \left(\frac{n \alpha}{n-2}|w|^{\frac{n}{n-2}}\right) \frac{d t}{t^{1+\frac{n \alpha}{n-2}}}
$$

where $\alpha=\beta+1<1$. The condition $\int_{B_{R}}|\Delta v|^{n / 2} d x \leq 1$ is equivalent to

$$
\int_{1}^{\infty}\left|w^{\prime \prime}(s)\right|^{\frac{n}{2}} s^{n-1} d s \leq 1
$$

where $w(t)=\int_{1}^{t} \int_{z}^{\infty}\left|w^{\prime \prime}(s)\right| d s d z$. Then, by Lemma 4.2,

$$
\int_{1}^{\infty} \exp \left(r|w|^{\frac{n}{n-2}}(t)\right) \frac{d t}{t^{r+1}} \leq C_{0},
$$

where $C_{0}=C_{0}(n, r)$. Set $r=\frac{n \alpha}{n-2}$, then from (4.6) and (4.7), we get

$$
\int_{B_{R}} \exp \left(b_{n, 2} \alpha|v|^{\frac{n}{n-2}}\right)|x|^{n \beta} d x \leq C_{0} .
$$

Next, we prove the sharpness of the constant $b_{n, 2} \alpha$. We consider the following set:

$$
\mathcal{B}=\left\{b: \exists C_{0}, \int_{B_{1}} e^{b|v|^{\frac{n}{n-2}}}|x|^{n \beta} d x \leq C_{0}, \forall v \in C_{c}^{\infty}\left(B_{1}\right) \text { and }\|\Delta v\|_{n / 2}=1\right\} .
$$


Then, (4.8) implies $b_{n, 2} \alpha \in \mathcal{B}$. Let $u$ be a positive function with support in the unit ball $B_{1}$ and equals 1 in $B_{r}$ with $0<r<1$. Let $b \in \mathcal{B}$, then

$$
C_{0} \geq r^{n \alpha} \exp \left(b /\|\Delta u\|_{n / 2}^{\frac{n}{n-2}}\right) .
$$

This leads to

$$
b \leq \alpha n \lim _{r \rightarrow 0} C_{2, \frac{n}{2}}\left(B_{r} ; B_{1}\right)^{\frac{2}{n-2}}\left(\log \frac{1}{r}\right),
$$

where $C_{2, \frac{n}{2}}\left(B_{r} ; B_{1}\right)=\inf \|\Delta u\|_{\frac{n}{2}}^{\frac{n}{2}}$ and the infimum is taken over all $u \in C_{0}^{\infty}(B)$ such that $u=1$ on $B_{r}$. By [Ada88, the right hand side of (4.9) is just $b_{n, 2} \alpha$. Therefore, $b_{n, 2} \alpha=\sup \mathcal{B}$.

Our goal is to apply Theorem 4.3 to obtain estimates on conic 4-manifolds. In the rest of this section, we work in dimension 4. Since Talenti's principle is also proved originally in the form of Newton potential Tal76, from the same argument in Theorem 4.3, the next corollary is easily followed:

Corollary 4.4. Suppose that $\Omega \subset \mathbb{R}^{4}$ is a bounded domain, $f \in C_{c}(\Omega)$ and $\|f\|_{L^{2}(\Omega)} \leq 1$. Let $u$ be the Newton potential of $f$, i.e.

$$
u=\int_{\Omega} \Gamma(x-y) f(y) d y,
$$

where $\Gamma(x)=\frac{1}{4 \pi^{2}|x|^{2}}$. Then we have that

$$
\int_{\Omega} \exp \left(32 \pi^{2}(1+\beta) u^{2}\right)|x|^{4 \beta} d x \leq C .
$$

Using the Green's function of the Laplacian on a 4-manifold, Corollary 4.4 allows us to obtain estimates in a neighborhood of a conic point. Following BCY92, we are able to prove the following global estimates:

Corollary 4.5. Suppose that $\left(M, g_{0}, D, g_{D}\right)$ is a conic 4-manifolds with $D=$ $\sum_{i=1}^{k} p_{i} \beta_{i}$ and $\beta_{1}=\min \left\{\beta_{i}\right\}$. Let $g_{1}=g_{D}$ and $d V_{i}$ be the volume elements of $g_{i}, i=0,1$. Let $\bar{u}=f_{M} u d V_{1}$. Then for any $u \in H^{2}\left(d V_{1}\right)$

$$
\log \int_{M} \exp (4|u-\bar{u}|) d V_{1} \leq C+\frac{1}{8 \pi^{2}\left(1+\beta_{1}\right)}\|\Delta u\|^{2},
$$

where $C=C(M)$ is a constant.

Proof. Since the Green's function $G(x, y)$ of $\Delta$ exists on smooth manifold $\left(M, g_{0}\right)$, we have

$$
u(x)-\tilde{u}=\int_{M} \Delta u(y) G(x, y) d V_{0}(y),
$$

where $\tilde{u}=\frac{1}{V_{0}(M)} \int_{M} u(y) d V_{0}(y)$. Note

$$
G(x, y)=\frac{1}{4 \pi^{2}} r^{-2} g(r)+H(x, y)
$$

for $H$ a bounded function on $M \times M, r=d(x, y)$ and $g(r)$ a function with support in $B_{r}$ with radius smaller than the injective radius of $\left(M, g_{0}\right)$. First, suppose that

$$
f(y)=\Delta u(y),\|f\|_{2} \leq 1 .
$$


Let $d V_{1}=\rho(x) d V_{0}=e^{4 \gamma(x)} d V_{0}$ be the volume element for the singular metric. Consider the following PDE on $M$

$$
\Delta \psi(x)=\frac{1}{V_{1}(M)}\left(\rho(x)-\frac{V_{1}(M)}{V_{0}(M)}\right) .
$$

(4.11) has a weak solution $\psi(x) \in W^{2, p}$ for $1<p<-\frac{1}{\beta_{1}}$. By the Sobolev embedding theorem , $\psi(x) \in L^{\frac{4 p}{4-2 p}}\left(d V_{0}\right) \subset L^{2}\left(d V_{0}\right)$. Let $\bar{u}=\frac{1}{V_{1}} \int_{M} u(y) d V_{1}(y)$. Then we have

$$
u-\bar{u}=\int_{M} f(y)(G(x, y)-\psi(y)) d V_{0}(y) .
$$

By Hölder's inequality, we have

$$
\left|\int_{M} f(y) \psi(y) d V_{0}\right| \leq\|f\|_{2}\|\psi\|_{2} .
$$

Combining (1.6), (4.13) and (4.10), we have

$$
|u(x)-\bar{u}| \leq\left|\frac{1}{4 \pi^{2}} \int_{B_{\delta}(x)} f(y) r^{-2} d V_{0}(y)\right|+C\|f\|_{2},
$$

where $\delta$ is the injective radius and $C=C\left(g_{1}, g_{0}\right)=\|\psi\|_{2}$. Pick a normal coordinates around $x$. The metric $g_{i j}(y)=\delta_{i j}+O\left(|y|^{2}\right)$. Then we see that

$$
\begin{aligned}
\left|\frac{1}{4 \pi^{2}} \int_{B_{\delta}(x)} f(y) r^{-2} d V_{0}(y)\right| & =\left|\frac{1}{4 \pi^{2}} \int_{B_{\delta}(x)} f(y) r^{-2}\left(1+O\left(r^{2}\right)\right) d y\right| \\
& \leq\left|\frac{1}{4 \pi^{2}} \int_{B_{\delta}(x)} f(y)\right| x-\left.y\right|^{-2} d y \mid+C\|f\|_{2} .
\end{aligned}
$$

Note that we may assume that $f$ has compact support in $B_{\delta}(x)$, because the integral over the rest part of the manifolds can be controlled by the $L^{2}$ norm of $f$. Let $u_{1}(x)=\frac{1}{4 \pi^{2}} \int_{\mathbb{R}^{4}} f(y)|x-y|^{-2} d y$. Then

$$
|u-\bar{u}| \leq\left|u_{1}\right|+C\|\Delta u\|_{2} .
$$

Suppose that $x=p_{i}$ with index $\beta_{i}$. By Corollary 4.4, we have

$$
\int_{B_{\delta}(x)} \exp \left(32 \pi^{2} \alpha_{i}\left(u_{1}\right)^{2}\right)|z|^{4 \beta_{i}} d z \leq c_{0},
$$

where $\alpha_{i}=\left(1+\beta_{i}\right)$. If $v$ is a non-constant function on $M$, by mean value inequality and (4.16) we have

$$
\begin{aligned}
\int_{B_{\delta}(x)} \exp (4|v(z)|)|z|^{4 \beta_{i}} d z & \leq \int_{B_{\delta}(x)} \exp \left(\frac{32 \pi^{2} \alpha_{i} v^{2}}{\|\Delta v\|_{2}^{2}}+\frac{1}{8 \pi^{2} \alpha_{i}}\|\Delta v\|_{2}^{2}\right)|z|^{4 \beta_{i}} d z \\
& \leq c_{0} \exp \left(\frac{\|\Delta v\|_{2}^{2}}{8 \pi^{2} \alpha}\right) .
\end{aligned}
$$

Let $\alpha=1+\beta_{1}=\min \left\{1+\beta_{i}\right\}$. Combining (4.15) and (4.17), we obtain in $B_{\delta}$;

$$
\int_{B_{\delta}(x)} \exp (4|u(z)-\bar{u}|) \rho(z) d V_{0} \leq C \exp \left(\frac{\left.\|\Delta u\|^{2}\right)}{8 \pi^{2} \alpha}\right) .
$$


On $M-\cup_{i} B_{\delta}\left(p_{i}\right)$, using partition of unity, we can assume that $u(z)-\bar{u}$ vanishes in $B_{\delta / 2}\left(p_{i}\right)$. Then we can apply Adams' inequality in the form of [BCY92] which gives

$$
\begin{aligned}
\int_{M-\cup B_{\delta / 2}\left(p_{i}\right)} \exp (4|u(z)-\bar{u}|) \rho(z) d V_{0} & \leq c_{0} \exp \left(\frac{\left.\|\Delta u\|^{2}\right)}{8 \pi^{2}}\right) \\
& \leq c_{0} \exp \left(\frac{\left.\|\Delta u\|^{2}\right)}{8 \pi^{2} \alpha}\right) .
\end{aligned}
$$

Thus, we combine (4.16) and (4.19) to get :

$$
\int_{M} \exp (4|u(z)-\bar{u}|) d V_{1} \leq C \exp \left(\frac{\left.\|\Delta u\|^{2}\right)}{8 \pi^{2} \alpha}\right) .
$$

This concludes the proof.

If the Paneitz operator $P$ is nonnegative with $\operatorname{Ker}(P)=\{$ constants $\}$, we may define the pseudo differential operator $\sqrt{P}$ and the Green's function of $\sqrt{P}$ has the same leading term as $-\Delta$. See Lemma 1.6 in CY95 for details. Then we can follow the proof in Corollary 4.5 to derive a lower bound of II on conic manifolds:

Theorem 4.6. Suppose that $\left(M, g_{0}, D, g_{D}\right)$ is a conic 4-manifolds with $D=\sum_{i=1}^{k} p_{i} \beta_{i}$ and $\beta_{1}=\min \left\{\beta_{i}\right\}$. Let $g_{1}=g_{D}$ and $d V_{i}$ be the volume elements of $g_{i}, i=0,1$. Let $\bar{u}=f_{M} u(y) d V_{1}(y)$. Let $P$ be the Paneitz operator of $g_{0}$ on $M$. Suppose that $P$ is nonnegative and $\operatorname{Ker}(P)=\{$ constants $\}$. Then for any $u \in H^{2}\left(d V_{1}\right)$

$$
\log \int_{M} \exp (4|u(x)-\bar{u}|) d V_{1}(x) \leq C+\frac{1}{8 \pi^{2}\left(1+\beta_{1}\right)} \int_{M} u(x) P u(x) d V_{0}(x),
$$

where $C=C\left(\beta_{1}, M\right)$ is a constant.

\section{Proof of Theorem 1.3}

The equation of constant $Q$-curvature (1.2) is the Euler-Lagrange equation of the II functional, which is studied in [BCY92] and CY95] for smooth metrics. Recall that

$$
\mathrm{II}_{g}(u)=\left\langle P_{g} u, u\right\rangle_{g}+2 \int_{M} Q u d V_{g}-\frac{k_{g}}{2} \log f_{M} \exp (4 u) d V_{g},
$$

where $k_{g}=\int_{M} Q d V_{g}$ and $\left\langle P_{g} u, u\right\rangle_{g}=\int_{M} u P_{g} u d V_{g}$. By a simple integration by part trick, $\left\langle P_{g} u, u\right\rangle_{g}$ may be well defined for $u \in H^{2}\left(d V_{g}\right)$. It is obvious that $\left\langle P_{g} u, u\right\rangle_{g}$ is conformally invariant. From now on, we simply write $\langle P u, u\rangle$ when no confusion arises.

Let $\left(M^{4}, g_{0}, D, g_{D}\right)$ be a conic manifold with $g_{D}(x)=e^{2 \gamma(x)} g_{0}(x)$. Let $g_{1}=g_{D}$ and $d V_{i}$ be the volume elements of $g_{i}, i=0,1$. The key estimate of BCY92, CY95. is to employ Adams' inequality Ada88 to derive a low bound of the II functional. By Adams' inequality and its modified form BCY92, CY95, if $u \in H^{2}\left(d V_{0}\right)$ and $\langle P u, u\rangle \leq 1$, we have that

$$
\int_{M} \exp \left(32 \pi^{2}|u(x)-\tilde{u}|^{2}\right) d V_{0}(x) \leq c_{0} V_{0}(M),
$$

where the mean value $\tilde{u}=f_{M} u d V_{0}$. In conic 4-manifolds, we use the modified Adams' inequality 4.6 to obtain the estimate for II functional. 
Proof of Theorem 1.3. By Theorem 4.6

$$
\log \left(\int_{M} \exp (4|u-\bar{u}|) d V_{1}\right) \leq \frac{1}{8 \pi^{2} \alpha}\langle P u, u\rangle+C(\alpha, M),
$$

where $\alpha=\left(1+\beta_{1}\right)$ and $\bar{u}=f_{M} u d V_{1}$. Mean value inequality implies:

$$
\begin{aligned}
\int_{M} Q_{1} u d V_{1} & =\int_{M} Q_{1}(u-\bar{u}) d V_{1}+\bar{u} k_{g_{1}} \\
& \leq \frac{1}{4 \epsilon} \int_{M} Q_{1}^{2} d V_{1}+\epsilon \int_{M}(u-\bar{u})^{2} d V_{1}+\bar{u} k_{g_{1}} .
\end{aligned}
$$

Here $k_{g_{1}}=k_{g_{0}}+8 \pi^{2} \sum_{i} \beta_{i}$ by Proposition 3.2, Notice that

$$
\|u-\bar{u}\|_{L^{2}\left(d V_{1}\right)} \leq\|u-\bar{u}\|_{H^{2}} \leq\langle P u, u\rangle,
$$

by Propositions 2.2 and 2.6. If $\bar{u}=0$, then (5.2), (5.3) and (5.4) give us the desired estimate:

$$
\begin{aligned}
\langle P u, u\rangle+2 \int_{M} Q_{1} u d V_{1} & -\frac{k_{g_{1}}}{2} \log \left(f_{M} \exp (4 u) d V_{1}\right) \\
\geq & \left(1-\frac{k_{g_{1}}}{16 \pi^{2} \alpha}-\epsilon\right)\langle P u, u\rangle+C(\alpha, \epsilon) .
\end{aligned}
$$

We have used Lemma 3.3 for the integrability of $Q_{1}^{2}$ in (5.5). If $k_{g_{1}}<16 \pi^{2} \alpha$, we can always choose an $\epsilon$ small enough such that $\frac{k_{g_{1}}}{16 \pi^{2} \alpha}+\epsilon<1$. Then (5.5) shows

$$
\mathrm{II}(u) \geq C^{\prime}\langle P u, u\rangle+C \geq C^{\prime \prime} .
$$

Let

$$
\Lambda=\inf \left\{\operatorname{II}(u): u \in H^{2}\left(d V_{1}\right), \int_{M} u d V_{1}=0\right\} .
$$

Take a minimizing sequence of II, namely $\left\{u_{i}\right\}_{i=1}^{\infty}$ such that $\mathrm{II}\left(u_{i}\right) \rightarrow \Lambda$ as $i \rightarrow \infty$ and $\int_{M} u_{i} d V_{1}=0$. By (5.6) we see that $\left\|\Delta u_{i}\right\|_{2}^{2}$ is bounded. Hence, $u_{i}$ is bounded in $H^{2}\left(d V_{1}\right)$ by Poincaré's inequality Proposition 2.6. Replaced by a subsequence, we may assume that $u_{i}$ converges weakly to some $w$ in $H^{2}\left(d V_{1}\right)$ and strongly to the same $w$ in $L^{2}\left(d V_{0}\right)$ by the compactness of the embedding, cf Proposition 2.5. Then, we claim that $w$ achieves the infimum.

Claim: $\operatorname{II}(w)=\Lambda$.

Proof of the claim. Since $u_{i}$ converges to $w$ weakly in $H^{2}\left(d V_{1}\right)$, we see that

$$
\langle P w, w\rangle \leq \liminf \left\langle P u_{i}, u_{i}\right\rangle
$$

and

$$
\int_{M} Q_{1} w d V_{1}=\lim _{i \rightarrow \infty} \int_{M} Q_{1} u_{i} d V_{1}
$$

In order to control the last term of II, we note that

$$
\begin{aligned}
\left|\exp \left(4 u_{i}\right)-\exp (4 w)\right| & =\left|\int_{u_{i}}^{w} 4 \exp (4 s) d s\right| \\
& \leq 4 \exp \left(4|w|+4\left|u_{i}\right|\right)\left|w-u_{i}\right|
\end{aligned}
$$


which leads to

$$
\begin{aligned}
\int_{M}\left|\exp \left(4 u_{i}\right)-\exp (4 w)\right| d V_{1} \leq & 4 \int_{M} \exp \left(4|w|+4\left|u_{i}\right|\right)\left|w-u_{i}\right| d V_{1} \\
\leq 4 & \left(\int_{M} \exp (4|4 w|)\right)^{\frac{1}{4}}\left(\int_{M} \exp \left(4\left|4 u_{i}\right|\right)\right)^{\frac{1}{4}} \\
& \cdot\left(\int_{M}\left|w-u_{i}\right|^{2}\right)^{\frac{1}{2}} .
\end{aligned}
$$

By Adams' Inequality, $\left(\int_{M} \exp (4|4 w|)\right)^{\frac{1}{4}}$ and $\left(\int_{M} \exp \left(4\left|4 u_{i}\right|\right)\right)^{\frac{1}{4}}$ are bounded. Then (5.7) shows that $\int_{M}\left|\exp \left(4 u_{i}\right)-\exp (4 w)\right| d V_{1} \rightarrow 0$ as $i \rightarrow \infty$. Hence

$$
\lim _{i \rightarrow \infty} \log \left(f_{M} \exp \left(4 u_{i}\right) d V_{1}\right)=\log \left(f_{M} \exp (4 w) d V_{1}\right) .
$$

Therefore, we have that $\operatorname{II}(w) \leq \liminf \operatorname{II}\left(u_{i}\right)=\Lambda$. By the definition of $\Lambda$, we have $\mathrm{II}(w)=\Lambda$.

We have established the existence of a minimizer $w$ of the functional II. $w$ satisfies the corresponding Euler-Lagrange equation:

$$
P_{g_{1}} w+Q_{1}=\frac{k_{g_{1}} e^{4 w}}{2 f_{M} e^{4 w} d V_{1}}
$$

Note that (5.8) is equivalent to $Q_{w}=c$ where $Q_{w}$ is the $Q$-curvature of metric $g_{w}=e^{2 w} g_{1}$. Therefore, $w$ is a weak solution of

$$
P_{g_{1}} w+Q_{1}=c \cdot e^{4 w} .
$$

We next prove the regularity of the solution in 5.9. Suppose we have a $H^{2}$ solution $w$ of $P_{g_{1}} w+Q_{1}=c \cdot e^{4 w}$. By a renormalization of the volume, we may assume that $c= \pm 1$ or 0 . We assume that $c=1$ and $w$ is a weak solution of $P_{g_{1}} w+Q_{1}=e^{4 w}$. The proofs for other cases are similar. Thus, for any $v \in H^{2}\left(d V_{1}\right)$, $w$ satisfies:

$$
\begin{aligned}
0 & =\int_{M} v P_{g_{1}} w d V_{1}+\int_{M}\left(Q_{1}-e^{4 w}\right) v d V_{1} \\
& =\int_{M} v P_{g_{0}} w \rho d V_{0}+\int_{M}\left(Q_{1}-e^{4 w}\right) v \rho d V_{0},
\end{aligned}
$$

where $\rho(x)=\frac{d V_{1}}{d V_{0}}=e^{4 \gamma(x)}$. Thus, $w$ is a weak solution of

$$
P_{g_{0}} w=e^{4 w} \rho-Q_{1} \rho
$$

in $H^{2}\left(d V_{0}\right)$. Let

$$
h(x)=e^{4 w(x)} \rho(x)-Q_{1}(x) \rho(x)-\operatorname{div}\left(A_{g_{0}} d w\right) .
$$

By Corollary 3.3. $Q_{1} \rho \in L^{p}\left(d V_{0}\right)$ for $1<p<2, \rho \in L^{q_{1}}\left(d V_{0}\right)$ for $1<q_{1}<\frac{1}{1-\alpha}$ and by Adams' inequality, $e^{4 w} \in L^{p}\left(d V_{0}\right)$ for any $p>1$. This implies that $e^{4 w} \rho \in$ $L^{q_{1}}\left(d V_{0}\right)$ for $1<q_{1}<\frac{1}{1-\alpha}$. Thus $h \in L^{q}\left(d V_{0}\right)$ for some $1<q<\min \left\{2, \frac{1}{1-\alpha}\right\}$. Let $z(x)=\Delta w$. Then $\Delta z=h(x)$ in weak sense, i.e.

$$
\int_{M} z \Delta v d V_{0}=\int_{M} h v d V_{0}
$$


for any $v(x) \in H^{2}\left(d V_{0}\right)$. Now let $\Gamma(x, y)$ be the Green's function for $\Delta$. Let

$$
H(x)=\int_{M} h(y) \Gamma(x, y) d V_{0} .
$$

The regularity theory of elliptic equations [GT98 shows that $H(x) \in W^{2, q}\left(d V_{0}\right)$ and $\Delta H(x)=h(x)$ a.e.. Therefore,

$$
z(x)=H(x)+\bar{z} \quad \text { a.e. },
$$

where $\bar{z}=\int_{M} z d V_{0}$. We apply the regularity theory of elliptic equations again to obtain $w \in W^{4, q}\left(d V_{0}\right)$. Since $W^{4, q}\left(d V_{0}\right)$ is the regular Sobolev space and $4 q>4$, so we embed the solution $w$ into Hölder spaces by Morrey's embeeding theorem:

$$
w \in C^{\tau}, \tau<\min \{2,4(1+\beta)\} .
$$

For any $x_{0} \in M$ and $x_{0} \neq p_{i}, i=1,2, \ldots, k$, there exists a small neighborhood $B_{2 \epsilon}\left(x_{0}\right)$ of $x_{0}$ such that $p_{i} \notin B_{2 \epsilon}\left(x_{0}\right)$. By CY95, Mal06, the Green's function $G(x, y)$ of $P_{g_{0}}$ with respect to $g_{0}$ exists and is smooth on $M \times M \backslash\{(x, x)\}$. Furthermore, $G(x, y)$ and its derivatives have the following asymptotic properties Mal06:

$$
\begin{gathered}
\left|G(x, y)-\frac{1}{8 \pi^{2}} \log \frac{1}{|x-y|}\right| \leq C, x \neq y, \\
\left|\nabla^{i} G(x, y)\right| \leq C_{i} \frac{1}{|x-y|^{i}}, i=1,2,3 .
\end{gathered}
$$

Here $C, C_{i}, i=1,2,3$ are some constants depend on $\left(M, g_{0}\right)$. The Green's function of $P_{g_{0}}$ gives the representation of $w$,

$$
w(x)-\bar{w}=\int_{M} G(x, y) P_{g_{0}} w(y) d V_{0}(y) .
$$

Let $f(x)=e^{4 w(x)} \rho(x)-Q_{1}(x) \rho(x) . \quad f(x)$ is clearly integrable and bounded in $B_{\epsilon}\left(x_{0}\right)$, which implies that $w(x) \in C^{3}\left(B_{\epsilon}\left(x_{0}\right)\right)$. Since $h(x) \in C^{1}\left(M \backslash\left\{p_{i}\right\}\right)$, we can apply the regularity theory to $\Delta z(x)=h(x)$ to show that $z(x) \in C_{l o c}^{2, \tau}\left(M \backslash\left\{p_{i}\right\}\right)$, which implies $w \in C_{l o c}^{4, \tau}\left(M \backslash\left\{p_{i}\right\}\right)$. The standard bootstrapping technique then yields $w \in C^{\infty}\left(M \backslash\left\{p_{i}\right\}\right)$.

Remark 5.1. From the regularity argument, we can see the number 2 in (5.11) is introduced by the $L^{2-\epsilon}$ integrability of $Q_{1} \rho$. This term disappears when the original metric is conformally flat. In other words, the solution is in $C^{\tau}(M)$ for any $\tau<4\left(1+\beta_{1}\right)$ if the metric is conformally flat.

\section{Radial Symmetric Solutions}

In this section, we consider radial symmetric solutions on conic 4 spheres with standard background metric. Let $x_{S}, x_{N}$ be the two antipodes on $S^{4}$ and $D=$ $\beta_{0} x_{S}+\beta_{1} x_{N}$. Let $\eta: \mathbb{R}^{4} \rightarrow S^{4}$ be the inverse of the stereographic projection from north pole. Then

$$
\eta^{*}\left(g_{0}\right)=e^{2 z(x)} d s^{2}
$$

where $g_{0}$ is the standard metric on sphere, $d s^{2}$ is the Euclidean metric, and

$$
z(x)=\log \frac{2}{|x|^{2}+1} .
$$

Note $\eta^{-1}$ maps $x_{N}$ to infinity and $x_{S}$ to 0 . Let $T=S^{3} \times \mathbb{R}$ be a cylinder. Suppose

$$
G: S^{3} \times \mathbb{R} \rightarrow \mathbb{R}^{4}, G(w, t)=e^{t} w .
$$


The composition map $\eta \circ G: T \rightarrow S^{4}$ gives the standard cylindrical coordinate. The Paneitz operator with product metric $g_{T}$ is the following:

$$
P_{T}=\left(\partial_{t}^{2}+\Delta_{S^{3}}\right)^{2}-4 \partial_{t}^{2} \text {. }
$$

A radial symmetric function on $\mathbb{R}^{4}$ depends only on $t$. The constant $Q$-curvature equation is

$$
v^{\prime \prime \prime \prime}(t)-4 v^{\prime \prime}(t)=c \cdot e^{4 v}
$$

We only consider the positive $Q$-curvature since $k_{g}=8 \pi^{2}\left(2+\beta_{0}+\beta_{1}\right)>0$. By adding a constant, we may normalize (6.1) to the following

$$
v^{\prime \prime \prime \prime}(t)-4 v^{\prime \prime}(t)=e^{4 v(t)} .
$$

If a symmetric conic metric has $D=\beta_{0} x_{S}+\beta_{1} x_{N}$, the corresponding $v(t)$ must have linear growth at $\pm \infty$. Thus, we have the following boundary conditions at $\pm \infty$ :

$$
\lim _{t \rightarrow-\infty} v^{\prime}(t)=1+\beta_{0}, \lim _{t \rightarrow+\infty} v^{\prime}(t)=-\beta_{1}-1 .
$$

We classify all solutions of (6.2) satisfying (6.3).

Define $x_{1}(t)=v^{\prime}(t), x_{2}(t)=x_{1}^{\prime}(t), x_{3}(t)=x_{2}^{\prime}(t)-4 x_{1}(t)$ and $x_{4}(t)=x_{3}^{\prime}(t)$. From (6.2), we get the following system

$$
\left\{\begin{array}{llll}
x_{1}^{\prime} & = & x_{2} & \\
x_{2}^{\prime}= & 4 x_{1} & & \\
x_{3}^{\prime}= & & & \\
x_{4}^{\prime}= & & & x_{4} \\
& & & 4 x_{1} x_{4}
\end{array}\right.
$$

Note that the positivity of $Q$-curvature implies that $x_{4}>0$ for all $t$. The corresponding solution for standard 4-sphere (differing by a renormalization) in (6.2) is given by

$$
v(t)=-\log \cosh t+\frac{1}{4} \log 6 .
$$

It corresponds to $X(t)=\left(x_{1}, x_{2}, x_{3}, x_{4}\right)^{T}$ with

$$
X(t)=\left(-\tanh t,-\operatorname{sech}^{2} t, 2 \tanh t\left(\operatorname{sech}^{2} t+2\right), 6 \operatorname{sech}^{4} t\right)^{T} .
$$

First, we establish the first integral of (6.4).

Proposition 6.1. We have the following first integral

$$
2 x_{2}^{2}-8 x_{1}^{2}-4 x_{1} x_{3}+x_{4}=c,
$$

or equivalently,

$$
2 x_{2}^{2}+\frac{1}{2} x_{3}^{2}-\frac{1}{2}\left(x_{2}^{\prime}\right)^{2}+x_{4}=c .
$$

where $c$ is a constant.

Proof. Two formulae are equivalent. Multiply $v^{\prime}(t)$ on both sides of (6.2) and integrate by parts, we get (6.7). To get (6.8), simply apply $4 x_{1}(t)=x_{2}^{\prime}(t)-x_{3}(t)$ to (6.7). 
Remark 6.2. It is clear that all fixed points of (6.4) lie on a line $(a, 0,-4 a, 0)$ for $a \in \mathbb{R}$. The first integral (6.7) hence indicates that if (6.4) has a bounded solution then

$$
\lim _{t \rightarrow \infty}\left|x_{1}(t)\right|^{2}=\lim _{t \rightarrow-\infty}\left|x_{1}(t)\right|^{2}=a^{2} .
$$

In other words, $\beta_{0}=\beta_{1}=|a|-1$ is a necessary condition for the existence of a solution for (6.2) with (6.3). This is also a special case of the general Pohazaev idenity.

Since (6.4) is invariant under the transformation $t \rightarrow t+c$, we need to fix the gauge. First, we consider a special case with the following initial data:

$$
x_{1}(0)=x_{3}(0)=0, x_{2}(0)=p, x_{4}(0)=q .
$$

Here $p<0$ and $q>0$. Such solution is symmetric with respect to $t=0$, i.e. $x_{1}(t)$ and $x_{3}(t)$ are odd functions while $x_{2}(t)$ and $x_{4}(t)$ are even functions. The constant $c$ in (6.7) and (6.8) is given by $c=2 p^{2}+q$. Note that the standard solution of 4 -sphere in (6.5) has $p=-1$ and $q=6$.

For a fix $p<0$, we define

$$
\mathcal{Q}=\left\{q>0: \forall t>0, x_{2}(t)<0 \text { with } x_{2}(0)=p, x_{4}(0)=q\right\} .
$$

We show that $q=\sup \mathcal{Q}$ will give the precise initial data in (6.9) so that the corresponding solution is desired. We first state some lemmas to show that $\mathcal{Q}$ is connected, nonempty, and bounded from above.

Lemma 6.3. (Monotonicity Lemma) If $x_{i}(t)$ and $y_{i}(t)$ are two solutions for the system (6.4) and $x_{i}(0) \geq y_{i}(0), i=1,2,3,4$, then $x_{i}(t) \geq y_{i}(t)$ for all $t>0$. The equality holds if and only if $x_{i}(0)=y_{i}(0), i=1,2,3,4$.

Proof. When $x_{i}(0)=y_{i}(0), i=1,2,3,4$, then obviously $x_{i}(t) \equiv y_{i}(t)$ for all $t$. Suppose that $x_{j}(0)>y_{j}(0)$, for some $j \in\{1,2,3,4\}$. Then by continuity, there exits a $\epsilon>0$ such that $x_{i}(t)>y_{i}(t)$ for $t \in(0, \epsilon), 1 \leq i \leq j$. In particular, $x_{1}(t)>y_{1}(t)$ and

$$
\left(\log x_{4}(t)\right)^{\prime}-\left(\log y_{4}(t)\right)^{\prime}=4\left(x_{1}(t)-y_{1}(t)\right)>0,
$$

for $t \in(0, \epsilon)$. Therefore, $x_{4}(t)>y_{4}(t)$ on $(0, \epsilon)$ and $x_{i}(t)>y_{i}(t)$ on $(0, \epsilon)$, for $i=1,2,3,4$. Let

$$
J=\left\{t>0: x_{i}(t)>y_{i}(t)\right\}, t_{0}=\inf \{t>0: t \notin J\} .
$$

If $t_{0}<\infty, x_{i}\left(t_{0}\right)>y_{i}\left(t_{0}\right)$ for $i=1,2,3$ and $x_{4}\left(t_{0}\right)=y_{4}\left(t_{0}\right)$. However,

$$
\left(\log x_{4}\right)^{\prime}\left(t_{0}\right)-\left(\log y_{4}\right)^{\prime}\left(t_{0}\right)=4\left(x_{1}\left(t_{0}\right)-y_{1}\left(t_{0}\right)\right)>0 .
$$

It is impossible because of the definition of $J$. This implies that $J=(0, \infty)$.

The next lemma shows that $\mathcal{Q}$ is not empty.

Lemma 6.4. If $4 p+q \leq 0$, then $x_{2}<0$ and $x_{2}(t) \rightarrow-\infty$ as $t \rightarrow \infty$.

Proof. If $4 p+q<0$, then $x_{2}(0)=-\frac{q}{4}<0, x_{2}^{\prime}(0)=0$, and $x_{2}^{\prime \prime}(0)=4 p+q<0$. Hence $x_{2}(t)<0$ and $x_{2}^{\prime}(t)<0$ for $t \in(0, \epsilon)$. Clearly, by (6.4),

$$
x_{2}^{\prime \prime \prime}(t)=4 x_{2}^{\prime}(t)+x_{4}^{\prime}(t),
$$

$x_{2}^{\prime \prime \prime}(t)$ is negative if $x_{2}^{\prime}(t)<0$. Therefore $x_{2}^{\prime}(t) \rightarrow-\infty$ and $x_{2}(t) \rightarrow-\infty$ as $t \rightarrow \infty$.

If $4 p+q=0$, then we have $x_{2}^{(4)}(0)=x_{4}^{\prime \prime}(0)=4 p q<0$. Thus, $x_{2}^{\prime}(t)<0$ for $t \in(0, \epsilon)$. We then follow the above argument to get the same result. 
Then we prove the boundedness of $\mathcal{Q}$ by a comparison argument.

Lemma 6.5. For each $p<0$, there is some $q>0$ such that $\exists T>0, x_{2}(t)>0$ for $t>T$.

Proof. We argue by contradiction. Suppose that on the contrary, for any $q>0$, $x_{2}(t)<0$ for all $t>0$. Then, $x_{1}(t)<0, x_{4}^{\prime}(t)<0$, for $t \in(0,+\infty)$ and

$$
x_{4}^{\prime \prime}(t)=4\left(x_{2}(t)+4 x_{1}^{2}(t)\right) x_{4}(t) \geq 4 x_{2}(t) x_{4}(t) .
$$

By (6.4) we may assume that $4 p+q>0$. Thus $x_{2}^{\prime}(t)>0$ for $t \in(0, \epsilon)$ where $\epsilon$ is small. Let $T>0$ be maximal such that $x_{2}^{\prime}(t) \geq 0$ for $t \in[0, T)$. Then, $x_{4}(t) \leq q$ and $x_{2}(t) \geq p$ for $t \in[0, T)$. Hence, we obtain direct estimates:

$$
\begin{aligned}
& x_{4}^{\prime \prime}(t) \geq 4 x_{2}(t) x_{4}(t) \geq 4 p q, \\
& x_{4}^{\prime}(t) \geq 4 p q t, \\
& x_{4}(t) \geq q+2 p q t^{2} .
\end{aligned}
$$

Since

we have

$$
x_{2}^{\prime \prime}(t)=4 x_{2}+x_{4} \geq 4 p+q+2 p q t^{2},
$$

$$
x_{2}^{\prime}(t) \geq(4 p+q) t+\frac{2}{3} p q t^{3} .
$$

(6.10) holds for all $t \in(0, T]$ especially for $t=T$. By (6.10), we see that

$$
T \geq \sqrt{\frac{4 p+q}{-\frac{2}{3} p q}}=\sqrt{\frac{4 \frac{p}{q}+1}{-\frac{2}{3} p}} .
$$

Another consequence of (6.10) is

$$
x_{2}(t) \geq p+\frac{t^{2}}{2}(4 p+q)+\frac{1}{6} p q t^{4} .
$$

Let $t_{1}<t_{2}$ be two positive roots of $p+\frac{t^{2}}{2}(4 p+q)+\frac{1}{6} p q t^{4}$. If there exists $t<T$ such that

$$
t_{1}^{2}=\frac{-\sqrt{(4 p+q)^{2}-\frac{8 p^{2} q}{3}}+(4 p+q)}{-\frac{2}{3} p q}<t^{2}<\frac{\sqrt{(4 p+q)^{2}-\frac{8 p^{2} q}{3}}+(4 p+q)}{-\frac{2}{3} p q}=t_{2}^{2},
$$

then $x_{2}(t)>0$, which contradicts with our assumption. Thus, it is sufficient to show the interval $\left(t_{1}, t_{2}\right) \cap(0, T) \neq \varnothing$ for large $q$. Let $z=\frac{p}{q}$. We see that

$$
\begin{gathered}
t_{1}^{2}=\frac{-\sqrt{(4 z+1)^{2}-\frac{8 p z}{3}}+(4 z+1)}{-\frac{2}{3} p} \rightarrow 0, \quad \text { as } q \rightarrow \infty, \\
\sqrt{\frac{4 p+q}{-\frac{2}{3} p q}}=\sqrt{\frac{4 z+1}{-\frac{2}{3} p}} \rightarrow \sqrt{-\frac{3}{2 p}}>0, \quad \text { as } q \rightarrow \infty .
\end{gathered}
$$

Hence, for large $q$,

$$
t_{1}<\sqrt{\frac{4 p+q}{-\frac{2}{3} p q}} \leq \min \left\{T, t_{2}\right\} .
$$

We have thus finished the proof. 
Finally, we prove that there are bounded solutions of (6.4), which establishes the existence part of Theorem 1.5.

Theorem 6.6. For any fixed $p<0$, there is a unique $q>0$ such that $4 p+q>0$ and the solution of system (6.4) is bounded for all $t$ with initial data $x_{1}(0)=x_{3}(0)=0$, $x_{2}(0)=p$ and $x_{4}(0)=q$.

Proof. Let

$$
\mathcal{Q}=\left\{q>0: \forall t>0, x_{2}(t)<0 \text { with } x_{2}(0)=p, x_{4}(0)=q\right\} .
$$

By Lemma 6.4 if $4 p+q \leq 0, q \in \mathcal{Q} \neq \varnothing$. By monotonicity lemma 6.3, $\mathcal{Q}$ is a connected set and by Lemma 6.5. $q_{0}=\sup \{q \in \mathcal{Q}\}<\infty$. We claim that $q_{0}$ is a choice such that the corresponding solution of (6.4) is bounded. Let $\left\{y_{i}(t), i=\right.$ $1,2,3,4\}$ be a solution of (6.4) with initial value:

$$
\left(y_{1}, y_{2}, y_{3}, y_{4}\right)(0)=\left(0, p, 0, q_{0}\right)
$$

We claim that $y_{2}(t) \rightarrow 0$ as $t \rightarrow \infty$. We prove this claim by excluding several cases.

Case 1. $\exists t_{0}>0$ such that $y_{2}(t)<0$ for $0 \leq t<t_{0}, y_{2}\left(t_{0}\right)=0$, and $y_{2}^{\prime}\left(t_{0}\right)>0$. Then, there is $t_{1}>t_{0}$ such that $y_{2}\left(t_{1}\right)>0$. Because our solutions are continuously dependent on the initial values, there is a $q^{\prime}<q_{0}$ such that a solution $z(t)$ with $z_{i}(0)=y_{i}(0), i=1,2,3, z_{4}(0)=q^{\prime}$ and $\left|z_{2}\left(t_{1}\right)-y_{2}\left(t_{1}\right)\right|<y_{2}\left(t_{1}\right) / 2$. Then $\left.z\left(t_{1}\right)\right\rangle$ $\frac{y_{2}\left(t_{1}\right)}{2}>0$ which contradicts the definition of $q_{0}$.

Case 2. $\exists t_{0}>0$ such that $y_{2}(t)<0$ for $0 \leq t<t_{0}, y_{2}\left(t_{0}\right)=0$, and $y_{2}^{\prime}\left(t_{0}\right)=0$. This can be ruled out since

$$
y_{2}^{\prime \prime}\left(t_{0}\right)=4 y_{2}\left(t_{0}\right)+y_{4}\left(t_{0}\right)>0,
$$

and $y_{2}\left(t_{0}\right)$ is a local minimum, which contradicts the assumption of $t_{0}$.

We conclude from the Case 1 and 2 that $y_{2}(t)<0$ for all $t>0$. Hence, $y_{1}(t)<0$ for all $t>0$.

Case 3. $\liminf _{t \rightarrow+\infty} y_{2}(t)=-\infty$.

Pick an increasing sequence $\left\{t_{k}\right\}$ such that $t_{k} \rightarrow \infty$ and $y_{2}\left(t_{k}\right) \rightarrow-\infty$ as $k \rightarrow$ $+\infty$. For each $k$, we assume further that $y_{2}(t)<-\varepsilon_{k}$ for some $\varepsilon_{k}$ on $\left(0, t_{k}\right)$. By the definition of $q_{0}$, there is a sequence $\left\{q_{i}\right\}$ such that $q_{i}>q_{0}$ and $q_{i} \rightarrow q_{0}$, and there is a sequence of solutions $\left\{x^{i}(t)\right\}_{i=1}^{\infty}$ with initial value $\left(0, p, 0, q_{i}\right)$ such that

$$
\left\|x_{j}^{i}(t)-y_{j}(t)\right\|_{\infty} \rightarrow 0, j=1,2,3,4,
$$

as $i \rightarrow \infty$ in any compact subset of $\mathbb{R}$. For $t \in\left(0, t_{k}\right]$, pick $i_{k}$ such that $\| x_{j}^{i_{k}}(t)-$ $y_{j}(t) \|_{\infty}<\varepsilon_{k}$. However, since $q_{i_{k}}>q_{0}$, there is $t_{i_{k}}^{*}$ such that $x_{2}^{i_{k}}\left(t_{i_{k}}^{*}\right)=0$. By mean value theorem, there is a $\tau_{i_{k}}>0$ such that $\left(x_{2}^{i_{k}}\right)^{\prime}\left(\tau_{i_{k}}\right)=0$ and $x_{2}^{i_{k}}\left(\tau_{i_{k}}\right) \leq y_{2}\left(t_{k}\right)$. By (6.8),

$$
\begin{aligned}
\left(y_{2}\left(t_{k}\right)\right)^{2} & \leq 2\left(x_{2}^{i_{k}}\right)^{2}-\frac{1}{2}\left(\left(x_{2}^{i_{k}}\right)^{\prime}\right)^{2}+\frac{1}{2}\left(x_{3}^{i_{k}}\right)^{2}+x_{4}^{i_{k}} \\
& =2 p^{2}+q_{i_{k}}<2 p^{2}+2 q_{0} .
\end{aligned}
$$

This contradicts with our assumption of Case 3 .

Case 4. $\lim _{t \rightarrow+\infty} y_{2}(t)=-c<0$.

In this case, $y_{1}(t)<-\frac{c}{2} t+b$ for some constant $b$. By (6.4),

$$
y_{4}^{\prime}(t)<\left(b-\frac{c}{2} t\right) y_{4}
$$


and

$$
y_{4}(t) \leq C \exp \left(b t-\frac{c}{4} t^{2}\right) .
$$

Therefore, $y_{3}(t)=\int_{0}^{t} y_{4}(s) d s+y_{3}(0)$ is bounded. Since $y_{2}^{\prime}(t)=4 y_{1}+y_{3}$, we see $y_{2}^{\prime}(t)<-\frac{c}{2} t+b_{1}$ for some constant $b_{1}$, which implies that $y_{2}$ is not bounded and cannot have negative limit. We have reached a contradiction with the assumption of Case 4.

Case 5. $\liminf _{t \rightarrow+\infty} y_{2}(t)=-c<0$ while $\limsup _{t \rightarrow+\infty} y_{2}(t)>-c$.

We pick a sequence $t_{n} \rightarrow+\infty$ such that $y_{2}\left(t_{n}\right) \rightarrow-c, y_{2}^{\prime}\left(t_{n}\right)=0$,

$$
y_{2}^{\prime \prime}\left(t_{n}\right)=4 y_{2}\left(t_{n}\right)+y_{4}\left(t_{n}\right) \geq 0
$$

which means $\lim _{n \rightarrow \infty} y_{4}\left(t_{n}\right) \geq 4 c$. But by (6.4),

$$
y_{4}^{\prime}(t)=4 y_{4}(t) y_{1}(t)<0
$$

implies that $y_{4}$ is monotone and $y_{4}(t) \geq 4 c$ for $t \rightarrow \infty$. However, by (6.4), $y_{3}(t)$ is then unbounded. By evaluating (6.7) at $t_{n}$, a contradiction is reached.

We summarize our discussion above. By ruling out cases 1 to 5 , we have proved that $y_{2}(t) \rightarrow 0$ as $t \rightarrow+\infty$.

We now prove that $y(t)$ is a bounded solution of (6.4). We split the proof into two cases.

Case 1, suppose that $y_{2}(t)$ oscillates as $t \rightarrow+\infty$, i.e. there exist $t_{k} \rightarrow \infty$ as $k \rightarrow \infty$ such that $y_{2}^{\prime}\left(t_{k}\right)=0$.

Since $y_{3}$ is monotone, (6.8) implies that $y_{3}$ is bounded. Furthermore, by (6.8), $y_{2}^{\prime}(t)$ is bounded. Then, $y_{2}^{\prime}(t)=4 y_{1}(t)+y_{3}(t)$ implies that $y_{1}(t)$ is bounded. Hence, we have proved the theorem for this case.

Case 2, if $y_{2}$ is increasing for big $t$, i.e. $y_{2}^{\prime}>0$ for $t>t^{*} \gg 0$.

By (6.4), we have

$$
\frac{d}{d t}\left(\frac{1}{2}\left(y_{2}^{\prime}(t)\right)^{2}-2 y_{2}^{2}(t)\right)>0, t>t^{*}
$$

Thus, for $t^{*}<t_{1}<t_{2}$.

$$
\frac{1}{2}\left(y_{2}^{\prime}\left(t_{2}\right)\right)^{2}-2 y_{2}^{2}\left(t_{2}\right)>\frac{1}{2}\left(y_{2}^{\prime}\left(t_{1}\right)\right)^{2}-2 y_{2}^{2}\left(t_{1}\right),
$$

We claim $\frac{1}{2}\left(y_{2}^{\prime}\left(t_{1}\right)\right)^{2}-2 y_{2}^{2}\left(t_{1}\right) \leq 0$ for $t_{1}>t^{*}$. If not, then for any $t_{1}>t^{*}$,

$$
\frac{1}{2}\left(y_{2}^{\prime}\left(t_{1}\right)\right)^{2}-2 y_{2}^{2}\left(t_{1}\right)>c>0 .
$$

Since $y_{2}(t) \rightarrow 0$, we may choose $t_{2}>t_{1}$ such that $\left|y_{2}(t)\right|<\frac{c}{2}$. Then, by (6.12) and (6.13), we have $\frac{1}{2}\left(y_{2}^{\prime}\left(t_{2}\right)\right)^{2}>\frac{c}{2}$. This shows that $y_{2}$ is at least linearly increasing, which contradicts with the fact that $y_{2} \rightarrow 0$. Hence, we have proved that

$$
\frac{1}{2}\left(y_{2}^{\prime}\left(t_{1}\right)\right)^{2}-2 y_{2}^{2}\left(t_{1}\right) \leq 0
$$

for $t_{1}>t^{*}$. (6.14) shows that $y_{2}^{\prime}$ and $y_{3}(t)$ are bounded. We use $y_{2}^{\prime}(t)=4 y_{1}(t)+$ $y_{3}(t)$ in (6.4) to show that $y_{1}(t)$ is also bounded. This finishes the proof of case 2 .

We have thus proved Theorem 6.6.

We now discuss the uniqueness part in Theorem 1.5

Theorem 6.7. Fix a constant in the right hand side of the first integral (6.7), the bounded solution to the system (6.4) is unique up to a translation(dilation) in $t$. 
Proof. By a translation, we may assume that $x_{1}(0)=0$. Suppose the initial data is given by

$$
\left(x_{1}, x_{2}, x_{3}, x_{4}\right)(0)=(0, a, b, c),
$$

where $c>0$ and the corresponding solution $z_{i}(t)$. Then $(-1)^{i} z_{i}(-t)$ with initial value $(0, a,-b, c)$ is also a solution of (6.4). Without loss of generality, we may assume $b>0$ and $2 a^{2}+c=2 p^{2}+q$. Suppose $y_{i}(t)$ is a bounded solution such that

$$
\left(y_{1}, y_{2}, y_{3}, y_{4}\right)(0)=(0, p, 0, q) \text {. }
$$

If $p \leq a<0$ then $q \leq c$. By Lemma 6.3. $z_{i}(t)>y_{i}(t)$ for $t>0$. Hence

$$
z_{2}(t)-y_{2}(t)>0,\left(z_{2}(t)-y_{2}(t)\right)^{\prime}>0
$$

Furthermore, since the inequalities in (6.15) are strict, by Lemma 6.3 if $y_{2}$ is bounded, then $z_{1}(t) \rightarrow \infty$. Thus, $z(t)$ can not be a bounded solution of (6.4). If $p>a$ then $q>c$, let $\bar{z}_{i}(t)=(-1)^{i} z_{i}(-t)$. Then still $\bar{z}_{i}(t)<y_{i}(t)$ for $t>0$. By the same argument, $\bar{z}_{1}(t) \rightarrow-\infty$ as $t \rightarrow \infty$. Hence, $z(t)$ can not be a bounded solution either. Thus, we have proved the theorem.

\section{Asymptotic Behavior}

In this section, we establish a local asymptotic expansion for solutions of (1.7) in $\mathbb{R}^{4}$. Let $\mathcal{P}_{m}$ be the space of homogeneous polynomials with degree $m$. The eigenvalues and eigenfunctions of Laplacian $\Delta$ on $\mathbb{R}^{4}$ are described as follows.

Lemma 7.1. (e.g. [LP87]) Suppose that $x \in \mathbb{R}^{4} r=|x|$. The eigenvalues of $r^{2} \Delta$ on $\mathcal{P}_{m}$ are

$$
\left\{\lambda_{j}=2 j(2+2 m-2 j): j=0,1 \ldots,[m / 2]\right\} .
$$

The eigenfunctions corresponding to $\lambda_{j}$ are the functions of the form $r^{2 j} u$, where $u \in \mathcal{P}_{m-2 j}$ is harmonic.

The above lemma indicates that if $a$ is not an eigenvalue, $\left(r^{2} \Delta-a\right)$ is invertible. The next lemma shows that with the presence of a singular weight, we may still solve the double Laplace equation in $\mathcal{P}_{m}$.

Lemma 7.2. Let $\beta \in \mathbb{R},-1<\beta<0$.

1. $\beta \neq-1 / 2$. For any polynomial $f(x)$, there is a polynomial $q(x)$ such that

$$
\Delta^{2}\left(q(x) r^{4 \beta+4}\right)=f(x) r^{4 \beta} .
$$

More generally, there is a collection of polynomials $\left\{q_{l}\right\}_{l=0}^{k}$ such that

$$
\Delta^{2}\left(\sum_{l=0}^{k} q_{l}(x) r^{4 \beta+4}(\log r)^{l}\right)=f(x) r^{4 \beta}(\log r)^{k} .
$$

2. $\beta=-1 / 2$. For any polynomial $f$ with degree $\leq 2$ with $f(x)=\sum a_{i j} x_{i} x_{j}+$ $\sum b_{i} x_{i}+c$, there exists a function

$$
q(x)=a_{0} r^{2}+\tilde{a}_{i j} x_{i} x_{j} \log r+\tilde{b}_{i} x_{i} \log r+\tilde{c} \log r,
$$

such that $\Delta^{2}\left(q(x) r^{2}\right)=f(x) r^{-2}$. In particular, $a_{0}$ vanishes if $\tilde{a}_{i i}=0$, $i=1,2,3,4$, and $\tilde{a}_{i j}=0$ if $\operatorname{deg} f<2$. There exist polynomials $q_{l}$ such that

$$
\Delta^{2}\left(r^{2} \sum_{l=0}^{k+1} q_{l}(x)(\log r)^{l}\right)=f(x) r^{-2}(\log r)^{k} .
$$

Proof. See the appendix. 
Suppose that $u$ is a desired weak solution of (1.7). We consider

$$
\Delta^{2} u(x)=e^{4 u(x)}, \quad x \in B_{1}(0) .
$$

Then by the regularity theory in the proof of Theorem 1.3, $u-\beta \log r \in C^{\infty}\left(B_{1}(0)-\right.$ $\{0\}) \cap C^{4 \beta+4-\epsilon}\left(B_{1}(0)\right)$ for any $\epsilon>0$. Here $\beta=\beta_{0}$. Let $w=u(x)-\beta \log r$. Then

$$
\Delta^{2} w(x)=e^{4 w(x)}|x|^{4 \beta} .
$$

Theorem 7.3. Suppose that $w$ is a solution of (7.4) in $B_{1}$ and $w \in C^{\infty}\left(B_{1}(0)-\right.$ $\{0\}) \cap C^{4+4 \beta-\epsilon}\left(B_{1}(0)\right), \forall \epsilon>0$. If

Case 1: $-\frac{k+1}{k+2}<\beta<-\frac{k}{k+1}$, for some $k=0,1,2, \cdots$, then

$$
w=\sum_{l=1}^{k+1} q_{l}(x) r^{4 l(\beta+1)}+\psi(x),
$$

where $\psi(x) \in C^{4, \gamma}$ and $q_{l}$ are polynomials.

Case 2: $\beta=-\frac{2 k}{2 k+1}$, for some $k=0,1,2, \cdots$, then

$$
w=\sum_{l=1}^{2 k+2} q_{l}(x) r^{4 l(\beta+1)}+\psi(x),
$$

where $\psi(x) \in C^{4, \gamma}$ and $q_{l}$ are polynomials.

Case 3: $\beta=-\frac{2 k-1}{2 k}$, for some $k=0,1,2, \cdots$, then

$$
w=\sum_{l=1}^{2 k} q_{l}(x) r^{4 l(\beta+1)} P_{l}(\log r)+\psi(x) .
$$

where $\psi(x) \in C^{4, \gamma}, q_{l}$ and $P_{l}$ are polynomials.

We break the proof into 3 cases according to the value of $\beta$.

Proof of Case 1, $-\frac{k+1}{k+2}<\beta<-\frac{k}{k+1}$. Since $w \in C^{4 \beta+4-\epsilon}$, there exists a polynomial $g_{0}$ with degree not exceeding 3 such that $\bar{w}:=w-g_{0}=o\left(r^{4 \beta+4-\epsilon}\right)$. Furthermore,

$$
\Delta^{2} \bar{w}=e^{4 g_{0}}\left(e^{4 \bar{w}}-1\right) r^{4 \beta}+e^{4 g_{0}} r^{4 \beta} .
$$

Since $e^{4 g_{0}}$ is smooth, we find a polynomial $\phi_{0}(x)$ such that

$$
\left(\phi_{0}(x)-e^{4 g_{0}(x)}\right) r^{4 \beta}=O\left(r^{\gamma}\right) \text {, }
$$

where $\gamma>0$. In fact, since $\beta \neq-\frac{1}{2}$, we pick $\gamma=4 \beta+4$. By Lemma 7.2 , there is a polynomial $q_{0}(x)$ such that

$$
\Delta^{2}\left(q_{0}(x) r^{4 \beta+4}\right)=\phi_{0}(x) r^{4 \beta} .
$$

Then, from (7.5), (7.6) and (7.7), we have

$$
\begin{aligned}
\Delta^{2}\left(\bar{w}-q_{0}(x) r^{4 \beta+4}\right) & =e^{4 g_{0}}\left(e^{4 \bar{w}}-1\right) r^{4 \beta}+\left(e^{4 g_{0}}-\phi_{0}\right) r^{4 \beta} \\
& =o\left(r^{8 \beta+4-\epsilon}\right)+O\left(r^{\gamma}\right) .
\end{aligned}
$$

If $\beta>-\frac{1}{2}$, then the right hand side of (17.8) is Hölder continuous and by the elliptic regularity theory, we have finished the proof.

If $\beta<-\frac{1}{2}$, let $w_{1}=\bar{w}-q_{0}(x) r^{4 \beta+4}$. Then

$$
\Delta^{2} w_{1} \in L^{p}, 1<p<-\frac{4}{4+8 \beta} .
$$


By the elliptic regularity theory, $w_{1} \in W^{4, p} \hookrightarrow C^{8 \beta+8-\epsilon}$. Then we start the iteration procedure. At each step $m \leq j \leq k$, suppose that there are functions $w_{m}, \bar{w}_{m}$, $s_{m}, Q_{m}, g_{m}, R_{m}, \xi_{m}, \phi_{m}^{l}(x)$ such that:

i). $w_{m} \in C^{4(m+1)(\beta+1)-\epsilon}$ admits the following expansion

$$
\begin{aligned}
w_{m} & =\bar{w}_{m-1}-\sum_{l=0}^{k} q_{m-1}^{l} r^{4(l+1)(\beta+1)} \\
& =\bar{w}_{m-1}-Q_{m-1}(x),
\end{aligned}
$$

where $Q_{m-1}(x)=\sum_{l=0}^{k} q_{m-1}^{l} r^{4(l+1)(\beta+1)}$ and $s_{m}=s_{m-1}+Q_{m-1}, s_{0}=0$.

ii). $g_{m}$ are polynomials with $\operatorname{deg}\left(g_{m}\right) \leq 3$, such that

$$
\bar{w}_{m}:=w_{m}-g_{m}=o\left(r^{4(m+1)(\beta+1)-\epsilon}\right) .
$$

iii). $\bar{w}_{m}$ satisfies:

$$
\begin{aligned}
\Delta^{2} \bar{w}_{m} & =e^{4 s_{m-1}}\left(e^{4\left(\bar{w}_{m}+g_{m}+\sum_{l=0}^{k} q_{m-1}^{l} r^{4(l+1)(\beta+1)}\right)}-1\right) r^{4 \beta}+\xi_{m}(x) \\
& =e^{4 s_{m}}\left(e^{4 \bar{w}_{m}}-1\right) r^{4 \beta}+e^{4 s_{m-1}}\left[e^{4\left(g_{m}+\sum_{l=0}^{k} q_{m-1}^{l} r^{4(l+1)(\beta+1)}\right)}-1\right] e^{4 \beta}+\xi_{m}(x) \\
(7.12) & =o\left(r^{4[(m+2) \beta+m+1]-\epsilon}\right)+R_{m}(x)+\xi_{m}(x) .
\end{aligned}
$$

where

$$
R_{m}(x)=e^{4 s_{m-1}}\left[e^{4\left(g_{m}+\sum_{l=0}^{k} q_{m-1}^{l} r^{4(l+1)(\beta+1)}\right)}-1\right] e^{4 \beta},
$$

and $\xi_{m}(x)$ is a Hölder continuous functions. We observe that $R_{m}(x)$ can be expanded as

$$
R_{m}(x)=\sum_{l=0}^{k} \phi_{m}^{l}(x) r^{4[(l+1) \beta+l]}+O\left(r^{4[(k+2) \beta+k+1]}\right),
$$

where $\phi_{m}^{l}(x)$ are polynomials.

At step $j \leq k$, by Lemma 7.2 , for each $l$, there exist a polynomial $q_{j}^{l}(x)$ such that

$$
\Delta^{2}\left(q_{j}^{l}(x) r^{4(l+1)(\beta+1)}\right)=\phi_{j}^{l}(x) r^{4[(l+1) \beta+l]} .
$$

Let $Q_{j}(x)=\sum_{l} q_{j}^{l} r^{4(l+1)(\beta+1)}$ and $w_{j+1}=\bar{w}_{j}(x)-Q_{j}(x)$. Then by (7.11) and (17.14)

$$
\begin{aligned}
\Delta^{2} w_{j+1} & =o\left(r^{4[(j+2) \beta+j+1]-\epsilon}\right)+R_{j}(x)-\sum_{l=0}^{k} \phi_{j}^{l} r^{4[(l+1) \beta+l]}+\xi_{j} \\
& =o\left(r^{4[(j+2) \beta+j+1]-\epsilon}\right)+O\left(r^{4[(k+2) \beta+k+1]}\right)+\xi_{j} \\
& =o\left(r^{4[(j+2) \beta+j+1]-\epsilon}\right)+\xi_{j+1},
\end{aligned}
$$

where $\xi_{j+1}(x)$ is a Hölder continuous function. Let $s_{j+1}=s_{j}+Q_{j}$. If $j<k$, the right hand side of (7.15) is in $L^{p}$ for $1<p<-\frac{1}{(j+2) \beta+j+1}$. Thus $w_{j+1} \in W^{4, p}$ by the elliptic regularity theory and hence $w_{j+1} \in C^{4(j+2)(\beta+1)-\epsilon}$. Therefore, there exists a corresponding $g_{j+1}$ such that

$$
\bar{w}_{j+1}:=w_{j+1}-g_{j+1}=o\left(r^{4(j+2)(\beta+1)-\epsilon}\right) .
$$


Note $g_{j+1}$ has degree less than or equal to 3 . Then, by (7.11),

$$
\begin{aligned}
\Delta^{2} \bar{w}_{j+1} & =\Delta^{2} w_{j+1}=\Delta^{2}\left(\bar{w}_{j}-Q_{j}\right) \\
& =e^{4 s_{j}}\left(e^{4 \bar{w}_{j}}-1\right) r^{4 \beta}+e^{4 s_{j-1}}\left(e^{4\left(g_{j}+Q_{j-1}\right)}-1\right) r^{4 \beta}+\xi_{j}(x)-\sum_{l=0}^{k} \phi_{j}^{l}(x) r^{4[(l+1) \beta+l]} \\
& =e^{4 s_{j}}\left(e^{4 \bar{w}_{j}}-1\right) r^{4 \beta}+\left(R_{j}-\sum_{l=0}^{k} \phi_{j}^{l}(x) r^{4[(l+1) \beta+l]}\right) r^{4 \beta}+\xi_{j} \\
& =e^{4 s_{j+1}}\left(e^{4 \bar{w}_{j+1}}-1\right) r^{4 \beta}+e^{4 s_{j}}\left(e^{4\left(g_{j+1}+Q_{j}\right)}-1\right) r^{4 \beta}+O\left(r^{4[(k+2) \beta+k+1]}\right)+\xi_{j} \\
& =e^{4 s_{j+1}}\left(e^{4 \bar{w}_{j+1}}-1\right) r^{4 \beta}+R_{j+1}+\xi_{j+1} \\
& =o\left(r^{4[(j+3) \beta+j+2]-\epsilon}\right)+R_{j+1}+\xi_{j+1} .
\end{aligned}
$$

By expanding $\bar{w}_{j+1}$ as in (7.16), $R_{j+1}(x)$ is in the form of (7.13). If $j<k$, then we apply the above argument again for $m=j+1$. We iterate until $j=k$ when the right hand side of (7.15) is Hölder continuous. Thus, by the elliptic regularity theory, $w_{k+1} \in C^{4, \gamma}$ for some $\gamma>0$. This clearly gives the asymptotic expansion.

Proof of Case 2, $\beta=\frac{1}{2 k+1}-1$. This case is similar to Case 1 with minor changes. We assume that $k \geq 1$. The beginning steps are the same as in the first case. We use iteration. In this case, we assume that $w_{1} \in C^{8 \beta+8}$. If $\beta=\frac{1}{2 k}-1$, the remaining term like (7.12) has the following form

$$
R_{1}(x)=\phi_{0} r^{4 \beta}+\phi_{1} r^{8 \beta+4}+\cdots+\phi_{2 k-1} r^{8 k \beta+8 k-4}+\phi_{2 k}+O\left(r^{\gamma}\right)
$$

where $\phi_{l}$ are polynomials.

$$
4(l+1)(\beta+1)-4 \neq-2, l=0,1, \cdots, 2 k .
$$

So by Lemma 7.2, there exist polynomials $q_{1}^{l}(x)$ and $Q_{1}(x)=\sum_{l} q_{1}^{l}(x) r^{4(l+1)(\beta+1)}$ such that

$$
\Delta^{2} Q_{1}(x)=\sum_{l=0}^{2 k} \phi_{l}(x) r^{4(l+1) \beta+4 l} .
$$

The iteration procedures are almost the same as in Case 1. Note, however, at the final step, when $j=2 k-1$. By (7.16),

$$
\Delta^{2} w_{2 k}=o\left(r^{-\epsilon}\right)+\xi_{2 k}(x)
$$

and $w_{2 k} \in C^{4-\epsilon}$. Then there exists a degree 3 polynomial $g_{2 k}$ such that $\bar{w}_{2 k}=$ $w_{2 k}-g_{2 k} \in C^{4-\epsilon}$. Note that

$$
\begin{aligned}
\Delta^{2} \bar{w}_{2 k} & =e^{4 s_{2 k-1}}\left(\exp \left(4 \bar{w}_{2 k}+g_{2 k}+Q_{2 k-1}\right)-1\right) r^{4 \beta}+\xi_{2 k-1} \\
& =e^{4 s_{2 k}}\left(e^{4 \bar{w}_{2 k}}-1\right) r^{4 \beta}+r^{4 \beta} e^{4 s_{1}}\left(e^{Q_{2 k-1}}-1\right)+\xi_{2 k-1} \\
& =o\left(r^{4+4 \beta-\epsilon}\right)+R_{2 k}+\xi_{2 k-1} .
\end{aligned}
$$

We have the expansion

$$
R_{2 k}=\sum_{l=0}^{2 k} \phi_{l}^{2 k} r^{4((l+1) \beta+l)}+O\left(r^{\frac{4}{2 k+1}}\right)
$$


Solve

$$
\Delta^{2} Q_{2 k}=\sum_{l=0}^{2 k} \phi_{l}^{2 k} r^{4((l+1) \beta+l)},
$$

by Lemma 7.2 Let $w_{2 k+1}=\bar{w}_{2 k}-Q_{2 k}$. By (7.18)

$$
\Delta^{2} w_{2 k+1}=o\left(r^{4+4 \beta-\epsilon}\right)+O\left(r^{\frac{4}{2 k+1}}\right)+\xi_{2 k-1} .
$$

Hence, the right hand side of (7.17) is Hölder continuous. By the regularity theory of elliptic equations, $w_{2 k+1} \in C^{4, \gamma}$ for some $\gamma>0$ and we conclude the proof.

Proof of Case 3, $\beta=\frac{1}{2 k}-1$. As in previous cases, we replace $w$ by $\bar{w}=w-g_{0}(x)$ such that $\bar{w}=o\left(r^{4 \beta+4-\epsilon}\right)$.

If $k=1$, we use Taylor expansion of $e^{4 g_{0}(x)}$ at 0 to get

$$
\left(e^{4 g_{0}(x)}-1\right)=f(x) r^{-2}+O(1),
$$

where $f(x)$ is a polynomial with degree at most 2 . Then

$$
\begin{aligned}
\Delta^{2} \bar{w} & =e^{4 \bar{w}+4 g_{0}} r^{4 \beta} \\
& =e^{4 g_{0}}\left(e^{4 \bar{w}}-1\right) r^{4 \beta}+\left(e^{4 g_{0}}\right) r^{-2} \\
& =o\left(r^{8 \beta+4-\epsilon}\right)+f(x) r^{4 \beta}+\xi(x) \\
& =o\left(r^{-\epsilon}\right)+f(x) r^{-2}+\xi(x)
\end{aligned}
$$

where $\xi$ is a smooth function. As in (7.7), there exists a function $q_{0}(x)$ by (17.2) such that

$$
\Delta^{2}\left(q_{0} r^{2}\right)=f(x) r^{-2} .
$$

By (7.19) and (7.20),

$$
\Delta^{2}\left(\bar{w}-q_{0}(x) r^{2}\right)=o\left(r^{-\epsilon}\right)+\xi(x) .
$$

Apply the elliptic regularity theory to (7.21), we have

$$
w_{1}=\bar{w}-q_{0}(x) r^{2} \in C^{4-\epsilon} .
$$

Suppose that $\bar{w}_{1}=w_{1}-g_{1}(x)$ such that $g_{1}$ is a polynomial with degree 3 and $\bar{w}_{1}=o\left(r^{4-\epsilon}\right)$. Then

$$
\begin{aligned}
\Delta^{2} \bar{w}_{1} & =\Delta^{2}\left(w_{1}\right)=\Delta^{2}\left(\bar{w}-q_{0} r^{2}\right) \\
& =e^{4 w} r^{-2}-f(x) r^{-2} \\
& =e^{4\left(g_{1}+q_{0} r^{2}+g_{0}\right)}\left(e^{4 \bar{w}_{1}}-1\right) r^{-2}+e^{4 g_{0}+4 g_{1}}\left(e^{4 q_{0} r^{2}}-1\right) r^{-2} \\
& +e^{4 g_{0}}\left(e^{4 g_{1}}-1\right) r^{-2}+\xi(x) \\
& =o\left(r^{2-\epsilon}\right)+c \log r+R_{1}(x)+\xi(x) .
\end{aligned}
$$

Here, the remainder term

$$
\begin{aligned}
R_{1}(x) & =e^{4 g_{0}}\left(e^{4 g_{1}}-1\right) r^{-2} \\
& =f_{1}(x) r^{-2}+O(r),
\end{aligned}
$$

where $f_{1}(x)$ is a quadratic polynomial. So

$$
\Delta^{2} \bar{w}_{1}=\xi_{1}(x)+c \log r+f_{1}(x) r^{-2},
$$

for some Hölder continuous $\xi_{1}$. Pick $c_{1} \in \mathbb{R}$ and $q_{1}(x)$ a function in (7.2) such that 


$$
\Delta^{2} c_{1} r^{4} \log r=c \log r-c_{2}
$$

and

We obtain that

$$
\Delta^{2}\left[q_{1}(x) r^{2}\right]=f_{1}(x) r^{-2}
$$

$$
\Delta^{2}\left(\bar{w}_{1}-c_{1} r^{4} \log r-q_{1}(r) r^{2}\right)=\xi_{2}(x),
$$

where $\xi_{2}$ is a Hölder continuous function. This shows that

$$
w=\left(a_{i j} x_{i} x_{j}+b_{i} x_{i}+c\right) r^{2} \log r+c_{1} r^{4} \log r+\psi(x),
$$

where $\psi(x) \in C^{4, \gamma}$ for $\gamma>0$. Particularly, it concludes the case where $\beta=-1 / 2$. We are done.

For $\beta \neq-1 / 2$, there exists $q_{0}(x)$ a polynomial satisfying (7.7). Then

$$
w_{1}=\bar{w}-q_{0}(x) r^{4 \beta+4} \in C^{8 \beta+8-\epsilon} .
$$

There exists a polynomial $g_{1}(x)$ such that $\bar{w}_{1}=w_{1}-g_{1}=o\left(r^{8 \beta+8-\epsilon}\right)$. We expand $\Delta^{2} \bar{w}_{1}$ in the form of (7.11). Note that the remaining term $R_{1}(x)$ in (7.12) has the following form:

$$
\begin{aligned}
R_{1}(x) & =\sum_{l=1}^{2 k} \phi_{l} r^{4 l(\beta+1)-4}+O\left(r^{\gamma}\right) . \\
& =\sum_{l=1}^{2 k-1} \phi_{l} r^{\frac{2 l}{k}-4}+\phi_{2 k}+O\left(r^{\gamma}\right) .
\end{aligned}
$$

In (7.22),$\phi_{k}(x) r^{\frac{2 k}{k}-4}=\phi_{k}(x) r^{-2}$. We assume $\operatorname{deg} \phi_{k} \leq 2$, since $p_{m}(x) r^{-2}$ is Hölder continuous for any homogeneous polynomial $p_{m} \in \mathcal{P}_{m}$ for $m \geq 3$. We can find polynomials $q_{1}^{l}(x)$ for $l \neq k$, such that

$$
\Delta^{2}\left(q_{1}^{l}(x) r^{4 l(\beta+1)}\right)=\phi_{l}(x) r^{4 l(\beta+1)-4} .
$$

There exists a function $q_{1}^{k}(x)$ in the form of (7.2) such that $\Delta^{2}\left(q_{1}^{k}(x) r^{2}\right)=\phi_{k} r^{-2}$. Let

$$
Q_{1}(x)=\sum_{l=1}^{2 k-1} q_{1}^{l}(x) r^{4 l(\beta+1)} .
$$

Let $w_{2}=\bar{w}_{1}-Q_{1}(x)$. Then

$$
\Delta^{2} w_{2}=o\left(r^{16 \beta+12-\epsilon}\right)+\xi_{2}(x) .
$$

We iterate as in the proof of Case 1. Suppose that $w_{l} \in C^{4(l+1)(\beta+1)}, l \leq j \leq 2 k$ and $w_{j}=\bar{w}_{j-1}-Q_{j-1}$, for

$$
Q_{j-1}=\sum_{l=1}^{2 k} q_{j-1}^{l} r^{4 l(\beta+1)} P_{j-1}^{l}(\log r),
$$

where $q_{j-1}^{l}$ and $P_{j-1}^{l}$ are polynomials. For $w_{j}$ there is a degree 3 polynomial $g_{j}$ such that $\bar{w}_{j}=w_{j}-g_{j}$ and $\bar{w}_{j}=o\left(r^{4(l+1)(\beta+1)-\epsilon}\right)$. Thus

$$
\Delta^{2} \bar{w}_{j}=o\left(r^{4(j+1)(\beta+1)-\epsilon}\right)+R_{j}(x)+\xi_{j-1}(x) .
$$

Here $R_{j}(x)$ can be written as

$$
R_{j}(x)=\sum_{l \neq k, 1 \leq l \leq 2 k} \phi_{j}^{l}(x) \bar{P}_{j}^{l}(\log r) r^{\frac{2 l}{k}-4}+\phi_{j}^{k}(x) r^{-2} \bar{P}_{j}^{k}(\log r)+O\left(r^{\gamma}\right),
$$


where $\phi_{j}^{l}, \bar{P}_{j}^{l}$ are polynomials. We may assume that deg $\phi_{j}^{k} \leq 2$ because $p_{m}(x) r^{-2} \bar{P}_{j}^{k}(\log r)$ is Hölder continuous for any homogeneous polynomial $p_{m} \in \mathcal{P}_{m}, m \geq 3$. By Lemma 7.2 we can find

$$
Q_{j}(x)=\sum_{l=1}^{2 k} q_{j}^{l}(x) P_{j}^{l}(\log r) r^{4 l(\beta+1)},
$$

such that

$$
\Delta^{2} Q_{j}=\sum_{l \neq k, 1 \leq l \leq 2 k} \phi_{j}^{l}(x) \bar{P}_{j}^{l}(\log r) r^{\frac{2 l}{k}-4}+\phi_{j}^{k}(x) r^{-2} \bar{P}_{j}^{k}(\log r) .
$$

Then by (7.23), (7.24) and (7.25),

$$
\Delta^{2}\left(\bar{w}_{j}-Q_{j}\right)=o\left(r^{4(j+2)(\beta+1)-4-\epsilon}\right)+\xi_{j}(x) .
$$

Let $w_{j+1}=\bar{w}_{j}-Q_{j}$. Then, $w_{j+1}$ is in $W^{4, p}$ for $1<p<-\frac{1}{(j+2) \beta+j+1}$. The iteration procedure does not stop until $j=2 k-1$ when the right hand side of (17.26) is Hölder continuous. Again, we use the elliptic regularity theory to show $w_{2 k} \in C^{4, \gamma}$ and the proof is complete.

\section{UNIQUENESS RESULT WITH 2 SINGULARITIES}

In this section, we give a proof of Theorem [1.6. Let $M=S^{4}$. Let $g_{0}$ be the standard metric on 4 -sphere. Let $\left(M, g_{0}, D, g_{1}\right)$ be the conic sphere with divisor $D=\beta_{0} p_{0}+\beta_{1} p_{1}$, then $\int_{M} Q_{g_{1}} d V_{g_{1}}=8 \pi^{2}\left(2+\beta_{0}+\beta_{1}\right)$ by Proposition 3.2. Note if $w$ is a solution on the sphere with divisor $D$, we have

$$
P_{S^{4}} w+6=3\left(2+\beta_{0}+\beta_{1}\right) e^{4 w} .
$$

Here we have normalized the equation such that the conic sphere has the same volume as that of a standard 4 -sphere. Let $\bar{k}_{g}=3\left(2+\beta_{0}+\beta_{1}\right)$. We only consider solutions such that

$$
w-\sum_{i=0,1} \eta_{i}(x) \beta_{i} \log \left|x-p_{i}\right| \in H^{2}\left(d V_{0}\right),
$$

where $p_{1}$ and $p_{2}$ are two points on the sphere and $\eta_{i}(x)$ are cut off functions in the neighborhood of $p_{i}$ in (1.3), $i=0,1$ respectively. By a conformal transform on the sphere, we may assume that $p_{1}$ and $p_{2}$ are antipodes $x_{S}$ and $x_{N}$, respectively. By a stereographic projection from $x_{N}$, we obtain the equation on $\mathbb{R}^{4}$

$$
\Delta^{2} u=\bar{k}_{g} e^{4 u}
$$

We state two lemmas that describe the asymptotic behavior of $u$.

Lemma 8.1. $\Delta u-2 \beta_{0} \frac{1}{|x|^{2}}=-\frac{\bar{k}_{g}}{4 \pi^{2}} \int_{\mathbb{R}^{4}} \frac{e^{4 u(y)}}{|x-y|^{2}} d y-C_{1}$ where $C_{1} \geq 0$ is a constant.

Lemma 8.2. $u-\beta_{0} \log |x|=-\frac{\bar{k}_{g}}{8 \pi^{2}} \int_{\mathbb{R}^{4}} \log \frac{|x-y|}{|y|} e^{4 u(y)} d y+C_{0}$ where $C_{0}$ is a constant. Besides, for any $\epsilon>0$ there is an $R_{\epsilon}$ such that

$$
\left(-2-\beta_{1}\right) \log |x| \leq u \leq\left(-2-\beta_{1}+\epsilon\right) \log |x|,
$$

for $|x| \geq R_{\epsilon}$.

For the proof of Lemma 8.1 and Lemma 8.2. see Lemma 2.1 - 2.5 in Lin98. We should mention that since we always assume that the solution $u$ comes from a $H^{2}$ function on $S^{4}, u$ satisfies assumptions in Lin's paper for both lemmas.

Now we derive an asymptotic expansion of $u$ at infinity. 
Lemma 8.3. Let $u$ be a solution of (8.1). Then, $u$ has the following asymptotic expansion as $\infty$ :

$$
u(x)=-\left(2+\beta_{1}\right) \log |x|+c+O\left(|x|^{-1}\right)
$$

and

$$
\left\{\begin{array}{l}
-\Delta u(x)=|x|^{-2}\left(a_{0}+\sum_{4 l\left(\beta_{1}+1\right)<1} a_{0, l}|x|^{-4 l\left(\beta_{1}+1\right)} P_{0, l}(-\log |x|)+\sum_{i} a_{i} x_{i}|x|^{-2}\right. \\
\left.+\sum_{4 l\left(\beta_{1}+1\right)<1} \sum_{i=1}^{4} a_{i, l} x_{i}|x|^{-4 l\left(\beta_{1}+1\right)-2} P_{i, l}(-\log |x|)\right)+O\left(|x|^{-4}\right), \\
-\frac{\partial}{\partial x_{i}} \Delta u(x)=a_{0} x_{i}|x|^{-4}+O\left(|x|^{-(3+\delta)}\right), \\
-\frac{\partial^{2}}{\partial x_{i} \partial x_{j}} \Delta u(x)=O\left(|x|^{-4}\right),
\end{array}\right.
$$

for large $|x|$, where $c, 0<\delta<4\left(\beta_{1}+1\right)$ and $a_{i, l}$ are constants and $P_{i, l}$ are polynomials. Note that $a_{0}=2\left(2+\beta_{1}\right)$ is positive.

Proof. Let $w(x)=u\left(\frac{x}{|x|^{2}}\right)-\left(2+\beta_{1}\right) \log |x|$. By Lemma 8.1 and Lemma 8.2, we see that $w(x)$ satisfies

$$
\begin{cases}\Delta^{2} w(x)=\bar{k}_{g} e^{4 w}|x|^{4 \beta_{1}} & \text { in } \mathbb{R}^{4}-\{0\} \\ |w(x)|=o(\log |x|) & \text { as }|x| \rightarrow 0 \\ |\Delta w|=o\left(|x|^{-2}\right) & \text { as }|x| \rightarrow 0 .\end{cases}
$$

Let $h(x)$ be a weak solution of

$$
\begin{cases}\Delta^{2} h(x)=\bar{k}_{g} e^{4 w(x)}|x|^{4 \beta_{1}} & \text { in } B_{1}, \\ h(x)=w(x) & \text { on } \partial B_{1}, \\ \Delta h(x)=\Delta w(x) & \text { on } \partial B_{1} .\end{cases}
$$

By Lemma 8.2, $e^{4 w(x)}|x|^{4 \beta_{1}}$ is in $L^{p}\left(B_{1}\right)$ for $\left(-\beta_{1}\right)^{-1}>p>1$. By the regularity theory of elliptic equations, $\Delta h(x) \in W^{2, p}\left(B_{1}\right)$ and $h(x) \in W^{4, p}\left(B_{1}\right)$ and hence $h(x) \in C^{4 \tau}$ for $0<\tau<\left(1+\beta_{1}\right)$. Now, let $q(x)=w(x)-h(x)$. Then it satisfies that

$$
\begin{cases}\Delta^{2} q=0 & \text { in } B_{1}-\{0\} \\ q=\Delta q=0 & \text { on } \partial B_{1}, \\ |q(x)|=o(\log |x|),|\Delta q|=o\left(|x|^{-2}\right) & \text { as }|x| \rightarrow 0 .\end{cases}
$$

Thanks to the asymptotic property, we can still apply maximum principle to $\Delta q$ which implies $\Delta q \equiv 0$ and similarly, $q \equiv 0$. Therefore, $w(x)=h(x)$.

Applying the asymptotic expansion in Theorem 7.3, the lemma follows immediately.

We apply the moving plane method to prove the radial symmetry of solutions with two conical singularities. Following the convention in the literature, see for example GNN79, CGS89, Lin98, let $\lambda \in \mathbb{R}, T_{\lambda}=\left\{\left(x_{1}, x_{2}, x_{3}, x_{4}\right): x_{1}=\lambda\right\}$, $\Sigma_{\lambda}=\left\{x: x_{1}>\lambda\right\}$, and $x^{\lambda}=\left(2 \lambda-x_{1}, x_{2}, x_{3}, x_{4}\right)$. In order to initiate the moving plane in $x_{1}$ direction, we need the following two lemmas.

Lemma 8.4. Let $v$ be a positive function defined in a neighborhood of infinity satisfying the asymptotic expansion (8.3). Then there exists $\bar{\lambda}$ and $R>0$ such that

$$
v(x)>v\left(x^{\lambda}\right)
$$

holds for $\lambda<\bar{\lambda},|x| \geq R$ and $x \in \Sigma_{\lambda}$. 
Lemma 8.5. Suppose $v$ satisfies the assumption of Lemma 8.4 and $v(x)>v\left(x^{\lambda_{0}}\right)$ for $x \in \Sigma_{\lambda_{0}}$. Assume $v(x)-v\left(x^{\lambda_{0}}\right)$ is superharmonic in $\Sigma_{\lambda_{0}}$. Then there exist $\epsilon>0, S>0$ such that the followings hold.

(i) $v_{x_{1}}>0$ in $\left|x_{1}-\lambda_{0}\right|<\epsilon$ and $|x|>S$.

(ii) $v(x)>v\left(x^{\lambda}\right)$ in $x_{1} \geq \lambda_{0}+\frac{\epsilon}{2}>\lambda$ and $|x|>S$

for all $x \in \Sigma_{\lambda}, \lambda \leq \lambda_{1}$ with $\left|\lambda_{1}-\lambda_{0}\right|<c \epsilon$, where $c=c\left(\lambda_{0}, v\right)$ is a small positive number.

Both lemmas are contained in the celebrated paper by Caffarelli-Gidas-Spruck CGS89. For the proofs, see Lemma 2.3 and 2.4 in CGS89. We should remind the readers that although our asymptotic expansion is not the exact form in the above paper, the leading terms are the same. Hence the argument in CGS89. can be applied.

Proof of Theorem [1.6. For any $\lambda \neq 0$, let $w_{\lambda}(x)=u(x)-u\left(x^{\lambda}\right)$ in $\Sigma_{\lambda}$. Then $w_{\lambda}(x)$ satisfies

$$
\begin{cases}\Delta^{2} w_{\lambda}=b_{\lambda}(x) w_{\lambda} & x \in \Sigma_{\lambda}, \\ w_{\lambda}=\Delta w_{\lambda}=0 & x \in T_{\lambda},\end{cases}
$$

where

$$
b_{\lambda}(x)=\bar{k}_{g} \frac{e^{4 u(x)}-e^{4 u\left(x^{\lambda}\right)}}{u(x)-u\left(x^{\lambda}\right)}>0 .
$$

By our assumption at 0 ,

$$
w_{\lambda}(x)=\beta_{0} \log |x|+O(1) .
$$

By Lemma 8.4 $-\Delta w_{\lambda}>0$ for $x \in \Sigma_{\lambda}, \lambda \leq \bar{\lambda}<0,|x|>R$. Since $v(x)=-\Delta u>0$, there is $\bar{\lambda}_{1} \leq \bar{\lambda}$ such that $v\left(x^{\lambda}\right)<v(x)$ for $|x|<R$ and $\lambda<\bar{\lambda}_{1}$. Hence

$$
-\Delta w_{\lambda}(x)>0
$$

in $\Sigma_{\lambda}$ for $\lambda \leq \bar{\lambda}_{1}$. We remark that the singularity of $u$ at 0 does not affect the computation. By Lemma 8.3, $\lim _{|x| \rightarrow \infty} w_{\lambda}(x)=0$. By (8.4) we can choose $\delta$ small such that $w_{\lambda}>0$ on the boundary $\partial B_{\delta}(0)$. Apply maximum principle in $\Sigma_{\lambda}-B_{\delta}(0)$ , we have $w_{\lambda}(x)>0$ in $\Sigma_{\lambda}-B_{\delta}(0)$ for $\lambda \leq \bar{\lambda}_{1}$. Then taking $\delta \rightarrow 0$, we have

$$
w_{\lambda}(x)>0, x \in \Sigma_{\lambda} \text {. }
$$

Let

$$
\lambda_{0}=\sup \left\{\lambda<0: v\left(x^{\mu}\right) \leq v(x), x \in \Sigma_{\mu} \text { for } \mu \leq \lambda\right\} .
$$

If $\lambda_{0}=0$ then we are done. Otherwise, we claim that

$$
u(x) \equiv u\left(x^{\lambda_{0}}\right)
$$

for $x \in \Sigma_{\lambda_{0}}$. This also implies that $\lambda_{0}=0$. We argue by contradiction. Suppose that $\lambda_{0}<0$ and $w_{\lambda_{0}} \not \equiv 0$ in $\Sigma_{\lambda_{0}}$. By continuity, $\Delta w_{\lambda_{0}} \leq 0(=-\infty$ at 0$)$ in $\Sigma_{\lambda_{0}}$. Since $w_{\lambda_{0}}(x) \rightarrow 0$ as $|x| \rightarrow \infty$, by strong maximum principle $w_{\lambda_{0}}>0$ in $\Sigma_{\lambda_{0}}$. Then we have

$$
\Delta^{2} w_{\lambda_{0}}=\bar{k}_{g}\left(e^{4 u}(x)-e^{4 u\left(x^{\lambda_{0}}\right)}\right)>0
$$

Hence $\Delta w_{\lambda_{0}}$ is subharmonic. Similar to (8.5), we apply strong maximum principle in $\Sigma_{\lambda_{0}}$ to get $\Delta w_{\lambda_{0}}(x)<0$ in $\Sigma_{\lambda_{0}}$. By the definition of $\lambda_{0}$, there is a sequence 
$\lambda_{n} \downarrow \lambda_{0}$ and $\lambda_{n}<0$ such that $\sup _{\Sigma_{\lambda_{n}}} \Delta w_{\lambda_{n}}>0$. Since $\lim _{|x| \rightarrow \infty} \Delta w_{\lambda_{n}}(x)=0$, there exists $z_{n} \in \Sigma_{\lambda_{n}}$ such that

$$
\Delta w_{\lambda_{n}}\left(z_{n}\right)=\sup _{x \in \Sigma_{\lambda_{n}}} \Delta w_{\lambda_{n}}(x)>0
$$

Note that clearly $z_{n} \neq 0$ and at each $z_{n}$,

$$
\nabla \Delta w_{\lambda_{n}}\left(z_{n}\right)=0 \text {. }
$$

By Lemma 8.5, $z_{n}$ are bounded. Suppose that $z_{0}$ is a limit point of $z_{n}$. $z_{0}$ can not be 0 since $\Delta w_{\lambda}(z) \rightarrow-\infty$ as $z \rightarrow 0$. If $z_{0} \in \Sigma_{\lambda_{0}}$, by continuity, $\Delta w_{\lambda_{0}}\left(z_{0}\right)=0$. This contradicts with the fact that $\Delta w_{\lambda_{0}}<0$ in $\Sigma_{\lambda_{0}}$. If $z_{0} \in T_{\lambda_{0}}$, then $\nabla\left(\Delta w_{\lambda_{0}}\left(z_{0}\right)\right)=0$, which along with (8.7) contradicts to Hopf's lemma at $z_{0}$. Hence the claim is proved.

By (8.6), we have

$$
u\left(x_{1}, x_{2}, x_{3}, x_{4}\right)=u\left(-x_{1}, x_{2}, x_{3}, x_{4}\right) .
$$

By choosing different coordinate systems, we get that $u$ is radial symmetric. We have thus proved Theorem 1.6 .

\section{APPENDIX}

Proof of Lemma 7.2. For each degree $m$, we only have to consider homogeneous polynomials in $\mathcal{P}_{m}$. We discuss 2 cases where $\beta \neq-\frac{1}{2}$ or $\beta=-\frac{1}{2}$.

Case 1. Suppose that $\beta \neq-\frac{1}{2}$. Let $p(x) \in \mathcal{P}_{m}$. By Euler formula, $x_{i} D_{i} p(x)=$ $m p(x)$.

$$
\Delta\left(p(x) r^{4 \beta+2}\right)=r^{4 \beta}\left(r^{2} \Delta p(x)+(4 \beta+2)(4 \beta+4+2 m) p(x)\right)
$$

So $\Delta\left(p(x) r^{4 \beta+2}\right)=f(x) r^{4 \beta}$ if

$$
r^{2} \Delta p(x)+(4 \beta+2)(4 \beta+4+2 m) p(x)=f(x) .
$$

Note if $\beta \in(-1,0)$ and $\beta \neq-1 / 2$, then $(4 \beta+2)(4 \beta+4+2 m)$ can not be an eigenvalue of $r^{2} \Delta$. Then by Lemma 7.1, we see that there exists a $p(x)$ such that

$$
\Delta\left(p(x) r^{4 \beta+2}\right)=f(x) r^{4 \beta} .
$$

Now let $q(x)$ be a homogeneous polynomial with degree $m$. Then

$$
\Delta\left(q(x) r^{4 \beta+4}\right)=r^{4 \beta+2}\left(r^{2} \Delta q(x)+(4 \beta+4)(4 \beta+6+2 m) q(x)\right)
$$

Apply Lemma 7.1 again, there is a polynomial such that $\Delta\left(q(x) r^{4 \beta+4}\right)=p(x) r^{4 \beta+2}$.

To solve

$$
\Delta^{2} g=f(x)(\log r)^{k}|x|^{4 \beta}
$$

we first compute

$$
\begin{aligned}
\Delta\left[p(x) r^{4 \beta+2} \log r\right] & =r^{4 \beta} \log r\left(r^{2} \Delta p(x)+(4 \beta+2)(2 m+4 \beta+4) p(x)\right) \\
& +r^{4 \beta} \phi(x),
\end{aligned}
$$

where $\phi(x) \in \mathcal{P}_{m-2}$. First, we can solve

$$
r^{2} \Delta p_{1}(x)+(4 \beta+2)(2 m+4 \beta+4) p_{1}(x)=f(x),
$$

as in (8.8). We can also find a polynomial $p_{2}(x)$ such that $\Delta\left(p_{2}(x) r^{4 \beta+2}\right)=\phi(x) r^{4 \beta}$. Thus,

$$
\Delta\left[r^{4 \beta+2}\left(p_{1} \log r-p_{2}\right)\right]=f(x) r^{4 \beta} \log r .
$$


By a similar argument, there exist $q_{1}, q_{2}$ such that

$$
\Delta\left[r^{4 \beta+4}\left(q_{1}(x) \log r+q_{2}(x)\right)\right]=\left(p_{1}(x)|x|^{2} \log r-p_{2}(x)|x|^{2}\right) r^{4 \beta},
$$

hence

$$
\Delta^{2}\left[\left(q_{1}(x) \log r+q_{2}(x)\right) r^{4 \beta+4}\right]=f(x)(\log r) r^{4 \beta} .
$$

This gives the solution $g=r^{4 \beta+4}\left(q_{1}(x) \log r+q_{2}(x)\right)$ for $k=1$ in (8.10). We use induction for $k \geq 2$. Suppose that we can find solutions of (17.1) for $0 \leq l \leq k-1$

$$
\begin{aligned}
\Delta\left(p(x) r^{4 \beta+2}(\log r)^{k}\right) & =r^{4 \beta}(\log r)^{k}\left(r^{2} \Delta p(x)+(4 \beta+2)(4 \beta+4+2 m) p(x)\right) \\
& +\sum_{j=0}^{k-1} r^{4 \beta} \phi_{j}(x)(\log r)^{j},
\end{aligned}
$$

where $\phi_{j}(x)$ are polynomials. Then we can find $p(x)$ such that

$$
r^{2} \Delta p(x)+(4 \beta+2)(4 \beta+4+2 m) p(x)=f(x) .
$$

In the remaining terms $\sum_{j=0}^{k-1} r^{4 \beta} \phi_{j}(x)(\log r)^{j}$, the degrees of $\log r$ are strictly smaller than $k$. Therefore, the remaining terms can be solved by induction and there exist $\left\{p_{l}(x)\right\}$ such that

$$
\Delta \sum_{l} p_{l} r^{4 \beta+2}(\log r)^{l}=f(x) r^{4 \beta}(\log r)^{k} .
$$

Repeat the argument for each $\tilde{f}(x)=p_{l}(x)|x|^{2}$ and we can solve (8.10).

Case 2. $\beta=-\frac{1}{2}$. If homogeneous degree $m=0$, we see that $\Delta^{2}\left(\frac{c}{16} r^{2} \log r\right)=$ $c r^{-2}$. So this is true for degree 0 polynomial. If $m=1$, direct computation shows that

$$
\Delta^{2}\left(\frac{a_{i} x_{i}}{48} r^{2} \log r\right)=a_{i} x_{i} .
$$

If $m=2$, we have for $i \neq j$,

$$
\Delta^{2}\left(x_{i} x_{j} r^{2} \log r\right)=96 x_{i} x_{j} r^{-2} .
$$

For $i=j$, we compute

$$
\Delta^{2}\left(x_{i}^{2} r^{2} \log r\right)=32+96 x_{i}^{2} r^{-2}+48 \log r .
$$

Note that $\Delta^{2}\left(r^{4} \log r\right)=7 \times 64+3 \times 128 \log r$. Since $\Delta^{2} r^{4}=192$, we can still find a solution for $\Delta^{2}\left(q(x) r^{2}\right)=x_{i}^{2} r^{2}$ in the form of (7.2).

For functions in the form of $f(x) r^{-2}(\log r)^{l}$ in (17.3), we argue by induction with respect to $k$. Note the above argument is for $l=0$. Suppose that for $0 \leq l \leq k-2$, we have a solution for (7.3). We prove for $l=k-1$. Any quadratic polynomial $f(x)$ is a linear combination of $1, x_{i}, x_{i} x_{j}, x_{i}^{2}$. Thus, we only have to consider these 4 subcases.

Subcase $1, f(x)=1$. Take test function $c r^{2}(\log r)^{k}$. Compute

$$
\Delta^{2}\left[c r^{2}(\log r)^{k}\right]=c r^{-2}\left(c_{1}(\log r)^{k-4}+c_{2}(\log r)^{k-3}+c_{3}(\log r)^{k-2}+2^{4}(\log r)^{k-1}\right),
$$

where $c_{i}$ are polynomials of $k$ and $c_{i}=0$ for $k=1,2, \ldots, 4-i$. Let $c=2^{-4}$ and the first term can be cancelled. The remaining terms $f-\Delta^{2}\left[c r^{2}(\log r)^{k}\right]$ in (8.13) are lower degrees terms and can be solved by induction. 
Subcase 2, $f(x)=x_{i}$. We consider a test function of the form $c x_{i} r^{2}(\log r)^{k}$. Direct computation shows

$$
\Delta^{2}\left(c x_{i} r^{2}(\log r)^{k}\right)=c k \frac{x_{i}}{r^{2}} \log ^{k-4}(r)\left(\sum_{i=0}^{2} c_{i}(\log r)^{i}+48 \log ^{3}(r)\right) .
$$

Likewise, take $c=(48 k)^{-1}$. Then, $f(x)-\Delta^{2}\left(c x_{i} r^{2}(\log r)^{k}\right)$ has lower degrees in $\log r$ and we can solve the remaining terms by induction.

Subcase $3, f(x)=x_{i} x_{j}, i \neq j$. By taking out a test function in the form of $c x_{i} x_{j} r^{2}(\log r)^{k}$ with a proper choice of $c$, we may reduce the top degree of $\log r$. Then, the lower degree terms can be solved by induction. The argument is similar to subcase 2 .

Subcase $4, f(x)=x_{i}^{2}$. We compute directly,

$$
\Delta^{2}\left(x_{i}^{2} r^{2}(\log r)^{k}\right)=x_{i}^{2} r^{-2}(\log r)^{k-1} k \times 96+x_{i}^{2} r^{-2} k P(\log r)+Q(\log r),
$$

where $P$ and $Q$ are polynomials in one variable and $\operatorname{deg} P(x) \leq k-2, \operatorname{deg} Q(x) \leq k$. Note that the degree of $P(x)$ is less than $k-1$. Thus, by induction assumption there exists a function $\bar{P}(x)$ in the form of (7.3) such that

$$
\Delta^{2}\left(\bar{P}(x) r^{2}\right)=x_{i}^{2} r^{-2} P(\log r) .
$$

For $Q(x)$ in (8.14), if it has degree $k$, there is a test function in the form of $c r^{4}(\log r)^{k}$. We compute

$$
\begin{aligned}
\Delta^{2}\left(r^{4}(\log r)^{k}\right) & =k \log ^{k-4}(r)((k-1)(k-2)(k-3) \\
& +b_{1} k(k-1)(k-2) \log (r) \\
& \left.+b_{2}(k-1) k \log ^{2}(r)+b_{3} k \log ^{3}(r)\right)+12 \times 16 \log ^{k}(r),
\end{aligned}
$$

where $b_{i}$ are constants independent of $k$. Suppose that $a_{k} \neq 0$ is the coefficient of the top degree term of $Q(x)$. Then,

$$
R(\log r)=Q(\log r)-\Delta^{2}\left(\frac{a_{k}}{12 \times 16} r^{4}(\log r)^{k}\right)
$$

is a polynomial in $\log r$ with degree less than $k$. Thus, we can apply the induction assumption to $R(\log r)$ to show that there exists a polynomial $\bar{Q}(x)$ with $\operatorname{deg} \bar{Q}(x) \leq$ $k$ and

$$
\Delta^{2}\left(r^{4} \bar{Q}(\log r)\right)=Q(\log r) .
$$

Now let

$$
\tilde{f}(x)=\frac{1}{96 k}\left(x_{i}^{2} r^{2}(\log r)^{k}-\bar{P}(x) r^{2}-r^{4} \bar{Q}(\log r)\right) .
$$

By (8.14), (8.15) and (8.17),

$$
\Delta^{2} \tilde{f}(x)=x_{i}^{2} r^{-2}(\log r)^{k-1}
$$

This completes the last case.

\section{REFERENCES}

[Ada88] David R Adams, A sharp inequality of $j$. moser for higher order derivatives, Annals of Mathematics 128 (1988), no. 2, 385-398.

[BCY92] Thomas P Branson, Sun-Yung A Chang, and Paul C Yang, Estimates and extremals for zeta function determinants on four-manifolds, Communications in mathematical physics 149 (1992), no. 2, 241-262. 
[BFR09] Paul Baird, Ali Fardoun, and Rachid Regbaoui, Prescribed q-curvature on manifolds of even dimension, Journal of Geometry and Physics 59 (2009), no. 2, 221 - 233.

[BN19] Reto Buzano and Huy The Nguyen, The chern-gauss-bonnet formula for singular noncompact four-dimensional manifolds, Communications in Analysis and Geometry $\mathbf{2 7}$ (2019), no. 8, 1697-1736.

[BØ91] Thomas P Branson and Bent Ørsted, Explicit functional determinants in four dimensions, Proceedings of the American Mathematical Society 113 (1991), no. 3, 669-682.

[Bre03] Simon Brendle, Global existence and convergence for a higher order flow in conformal geometry, Annals of mathematics 158 (2003), no. 1, 323-343.

[CC96] Jeff Cheeger and Tobias H Colding, Lower bounds on ricci curvature and the almost rigidity of warped products, Annals of mathematics 144 (1996), no. 1, 189-237.

$\left[\mathrm{CC}^{+} 97\right]$ Jeff Cheeger, Tobias $\mathrm{H}$ Colding, et al., On the structure of spaces with ricci curvature bounded below. i, Journal of Differential Geometry 46 (1997), no. 3, 406-480.

[CDS13] Xiuxiong Chen, Simon Donaldson, and Song Sun, Kähler-einstein metrics on fano manifolds. iii: Limits as cone angle approaches $2 \pi$ and completion of the main proof, Journal of the American Mathematical Society 28 (2013), no. 1, 235-278.

[CDS14] _ Kähler-einstein metrics on fano manifolds. $i$ : Approximation of metrics with cone singularities, Journal of the American Mathematical Society 28 (2014), no. 2, $138-146$.

[CDS15] _ Kähler-einstein metrics on fano manifolds. ii: Limits with cone angle less than 2 $\pi$, Journal of the American Mathematical Society 28 (2015), no. 1, 199-234.

[CGS89] Luis A Caffarelli, Basilis Gidas, and Joel Spruck, Asymptotic symmetry and local behavior of semilinear elliptic equations with critical sobolev growth, Communications on Pure and Applied Mathematics 42 (1989), no. 3, 271-297.

[CL95] Wenxiong Chen and Congming Li, What kinds of singular surfaces can admit constant curvature?, Duke Mathematical Journal 78 (1995), no. 2, 437-451.

[CY95] Sun-Yung A Chang and Paul C Yang, Extremal metrics of zeta function determinants on 4-manfilds, Annals of Mathematics 142 (1995), no. 1, 171-212.

[DM08] Zindine Djadli and Andrea Malchiodi, Existence of conformal metrics with constant q-curvature, Annals of Mathematics (2008), 813-858.

[FL16] Hao Fang and Mijia Lai, On convergence to a football, Mathematische Annalen 366 (2016), no. 1, 83-100.

[FW19] Hao Fang and Wei Wei, $\sigma_{2}$ yamabe problem on conic 4-spheres, Calculus of Variations and Partial Differential Equations 58 (2019), no. 4, 119

[GJMS92] C Robin Graham, Ralph Jenne, Lionel J Mason, and George AJ Sparling, Conformally invariant powers of the laplacian, $i$ : Existence, Journal of the London Mathematical Society 2 (1992), no. 3, 557-565.

[GNN79] B. Gidas, Wei Ming Ni, and L. Nirenberg, Symmetry and related properties via the maximum principle, Comm. Math. Phys. 68 (1979), no. 3, 209-243.

[GT98] David Gilbarg and Neil S Trudinger, Elliptic partial differential equations of second order, springer, 1998.

[Gur99] Matthew J. Gursky, The principal eigenvalue of a conformally invariant differential operator with an application to semilinear elliptic pde, Communications in Mathematical Physics 207 (1999), no. 1, 131-143.

[HLW20] Ali Hyder, Changshou Lin, and Juncheng Wei, The su(3) toda system with multiple singular sources, Pacific Journal of Mathematics 305 (2020), 645-666.

[HMM19] Ali Hyder, Gabriele Mancini, and Luca Martinazzi, Local and Nonlocal Singular Liouville Equations in Euclidean Spaces, International Mathematics Research Notices (2019), rnz149.

[Lin98] C-S Lin, A classification of solutions of a conformally invariant fourth order equation in rn, Commentarii Mathematici Helvetici 73 (1998), no. 2, 206-231.

[LL11] Nguyen Lam and Guozhen Lu, Sharp singular adams inequalities in high order sobolev spaces, Methods and Applications of Analysis 19 (2011).

[LLL12] Jiayu Li, Yuxiang Li, and Pan Liu, The q-curvature on a 4-dimensional riemannian manifold $(m, g)$ with $\int_{m} q d v_{g}=8 \pi^{2}$, Advances in Mathematics 231 (2012), no. 3, 2194-2223.

[LP87] John M Lee and Thomas H Parker, The yamabe problem, Bulletin of the American Mathematical Society 17 (1987), no. 1, 37-91. 
[LT92] Feng Luo and Gang Tian, Liouville equation and spherical convex polytopes.

[Maa16] Ali Maalaoui, Prescribing the q-curvature on the sphere with conical singularities, DYNAMICAL SYSTEMS 36 (2016), no. 11, 6307-6330.

[Mal06] Andrea Malchiodi, Compactness of solutions to some geometric fourth-order equations, Journal fur die reine und angewandte Mathematik (Crelles Journal) 2006 (2006), no. 594, 137-174.

[Ndi07] Cheikh Birahim Ndiaye, Constant q-curvature metrics in arbitrary dimension, Journal of Functional Analysis 251 (2007), no. 1, 1 - 58.

[Tal76] Giorgio Talenti, Elliptic equations and rearrangements, Annali della Scuola Normale Superiore di Pisa-Classe di Scienze 3 (1976), no. 4, 697-718.

[Tar12] Cristina Tarsi, Adams' inequality and limiting sobolev embeddings into zygmund spaces, Potential Analysis 37 (2012), no. 4, 353-385.

[Tia96] Gang Tian, Kähler-einstein metrics on algebraic manifolds, Lecture Notes in Math. 1646 (1996).

[Tia15] K-stability and kähler-einstein metrics, Communications on Pure and Applied Mathematics 68 (2015), no. 7, 1085-1156.

[Tro89] Marc Troyanov, Metrics of constant curvature on a sphere with two conical singularities, pp. 296-306, Springer Berlin Heidelberg, Berlin, Heidelberg, 1989.

[Tro91] _ Prescribing curvature on compact surfaces with conical singularities, Transactions of the American Mathematical Society 324 (1991), no. 2, 793-821.

14 MacLean Hall, University of Iowa, Iowa City, IA, 52242

E-mail address: hao-fang@uiowa.edu

E-mail address: biaoma@uiowa.edu 